\title{
Tramadol/paracetamol fixed-dose combination in the treatment of moderate to severe pain
}

This article was published in the following Dove Press journal:

Journal of Pain Research

28 August 2012

Number of times this article has been viewed

\author{
Joseph V Pergolizzi Jr $r^{1,2}$ \\ Mart van de Laar $^{3}$ \\ Richard Langford ${ }^{4}$ \\ Hans-Ulrich Mellinghoff ${ }^{5}$ \\ Ignacio Morón Merchante ${ }^{6}$ \\ Srinivas Nalamachu ${ }^{7,8}$ \\ Joanne O'Brien' \\ Serge Perrot ${ }^{10}$ \\ Robert B Raffa"I \\ 'Department of Medicine, Johns \\ Hopkins University, Baltimore, \\ MD, USA; ${ }^{2}$ Association of Chronic \\ Pain Patients, Houston, TX, USA; \\ ${ }^{3}$ Arthritis Center Twente (MST and \\ UT), Enschede, The Netherlands; \\ ${ }^{4}$ Anaesthetics Laboratory, \\ St Bartholomew's Hospital, London, \\ UK; ${ }^{5}$ Department of Endocrinology, \\ Diabetology and Osteology, \\ Kantonsspital St Gallen, St Gallen, \\ Switzerland; ${ }^{6}$ Centro de Salud \\ Universitario Goya, Madrid, Spain; \\ ${ }^{7}$ Kansas University Medical Center, \\ Kansas City, KS, USA; ${ }^{8}$ International \\ Clinic Research, Leawood, KS, USA; \\ ${ }^{9}$ Department of Pain Management, \\ Beaumont Hospital, Beaumont, Dublin, \\ Ireland; ${ }^{\circ}$ Service de Médecine Interne \\ et Consultation de la Douleur, Hôpital \\ Dieu, Paris, France; "'Department \\ of Pharmaceutical Sciences, Temple \\ University School of Pharmacy, \\ Philadelphia, PA, USA
}

Correspondence: Joseph V Pergolizzi Jr

NEMA Research, Inc, 840-I I I th

Avenue N, Naples, FL 34108-1877, USA

$\mathrm{Tel}+\mathrm{I} 2395973662$

Fax + I 2395977566

Email jpjmd@msn.com

\begin{abstract}
Pain is the most common reason patients seek medical attention and pain relief has been put forward as an ethical obligation of clinicians and a fundamental human right. However, pain management is challenging because the pathophysiology of pain is complex and not completely understood. Widely used analgesics such as nonsteroidal anti-inflammatory drugs (NSAIDs) and paracetamol (acetaminophen) have been associated with adverse events. Adverse event rates are of concern, especially in long-term treatment or at high doses. Paracetamol and NSAIDs are available by prescription, over the counter, and in combination preparations. Patients may be unaware of the risk associated with high dosages or long-term use of paracetamol and NSAIDs. Clinicians should encourage patients to disclose all medications they take in a "do ask, do tell" approach that includes patient education about the risks and benefits of common pain relievers. The ideal pain reliever would have few risks and enhanced analgesic efficacy. Fixed-dose combination analgesics with two or more agents may offer additive or synergistic benefits to treat the multiple mechanisms of pain. Therefore, pain may be effectively treated while toxicity is reduced due to lower doses. One recent fixed-dose combination analgesic product combines tramadol, a centrally acting weak opioid analgesic, with low-dose paracetamol. Evidence-based guidelines recognize the potential value of combination analgesics in specific situations. The current guideline-based paradigm for pain treatment recommends NSAIDs for ongoing use with analgesics such as opioids to manage flares. However, the treatment model should evolve how to use low-dose combination products to manage pain with occasional use of NSAIDs for flares to avoid long-term and high-dose treatment with these analgesics. A next step in pain management guidelines should be targeted therapy when possible, or low-dose combination therapy or both, to achieve maximal efficacy with minimal toxicity.
\end{abstract}

Keywords: NSAIDs, opioids, combination analgesics, moderate pain, severe pain, analgesics, tramadol/paracetamol

\section{Introduction}

Pain is the oldest medical problem and has been a challenge for doctors since the origin of humanity. While scientific and technological breakthroughs have improved care in many areas, eradicating diseases and advancing longevity, pain remains a global public health issue. The World Health Organization (WHO) has promoted and disseminated guidelines on pain management, ${ }^{1}$ advocated for the use of analgesics, including opioids, ${ }^{2}$ and encouraged national programs for palliative care and the relief of cancer pain. ${ }^{3,4}$ Pain relief has been put forward as a fundamental human right. ${ }^{5-8}$ The third international symposium on the Societal Impact of Pain held in May 2012 in Copenhagen has finalized a position paper, seeking that chronic pain be recognised as a disease by the governments of member states. ${ }^{9}$ Despite pharmacological advances 
and numerous guidelines or consensus documents to inform clinicians about the appropriate prescribing of analgesics, pain is often under-treated. ${ }^{10-12}$ Inadequate analgesia may have roots in social, political, legal, cultural, and religious considerations, as well as the fundamental knowledge, differences in health care systems, and variations in clinical practice. ${ }^{13-16}$ However, it remains the imperative of medical professionals to relieve pain as much as possible. ${ }^{17-19}$ Regardless of the social and political factors complicating analgesic therapy, not treating pain is not an option and has been described as a "moral outrage." 20

The European Study of the Epidemiology of Mental Disorders reported from a questionnaire (1659 respondents, all of whom were $\geq 75$ years of age) that pain was the most commonly reported problem in this population $(55.2 \%)$, far exceeding the rate of depression and anxiety $(11.6 \%) .{ }^{21}$ In Europe, it is estimated that $19 \%$ of the general population suffers from chronic pain. ${ }^{22}$ A hospital-based survey in Germany reported that over $80 \%$ of patients $(n=438)$ experienced pain in the previous 3 months and pain was the main reason for hospital admission in over $60 \%$ of the cases. ${ }^{23}$ In the USA, chronic pain affects more people every year than diabetes, heart disease, and cancer combined. ${ }^{24,25}$ Chronic pain can occur in patients of any age, but it is more common among older individuals. ${ }^{26}$ Inadequately treated persistent pain may be associated with a number of adverse outcomes in older people, including functional impairment, reduced mobility, falls, slower rehabilitation, decreased socialization, inadequate sleep, disturbed appetite, and changes in mood. ${ }^{27}$ Pain negatively affects quality of life, adversely affects families, may result in lost or diminished productivity for society, and places a large burden on the health care system. In the USA in 2002-2003, over US\$4 billion was spent on headacherelated care alone, and this did not include over-the-counter medications, self-treatment, and inpatient treatment. ${ }^{28}$ The total global health care burden related to all types of acute and chronic pain syndromes is difficult to assess.

Although pain management guidelines address specific types of pain, they frequently recommend nonsteroidal anti-inflammatory drugs (NSAIDs) in cases where tissue damage and inflammation are absent. Due to serious gastrointestinal, cardiovascular, and renal side effects, caution is recommended when using high-dose NSAIDs, particularly when taken long-term. ${ }^{27,29}$ The appropriate use of NSAIDs, paracetamol, opioid analgesics, or combination products in the chronic pain population remains a subject of ongoing research.

\section{Meeting details}

A consensus meeting attended by all authors of this publication was held on November 20, 2010 in Paris, France, to discuss the use of high-dose NSAIDs, high-dose paracetamol, or tramadol/paracetamol (as an example of fixed-dose combination analgesics) for the management of moderate to severe pain from different etiologies. Tramadol/Paracetamol is - to our knowledge - the only fixed-dosed combination product where the dual mode of action of tramadol and the analgesic synergy between the two compounds have been proven in both preclinical studies (mouse model) ${ }^{30,31}$ and companion human studies. ${ }^{32,33}$ Presentations by five of the authors were followed by a group discussion and review of pain management issues regarding these drug classes and available guidelines/recommendations based on the clinical experiences of the participants. A manuscript was drafted, additional articles were reviewed and incorporated, and a final consensus was adopted by the group.

\section{Pain management and underlying pain mechanisms}

Pain management is complex for many reasons. Chronic pain may be broadly classified into nociceptive (pain owing to tissue disease or damage, including inflammatory and visceral pain), neuropathic (pain caused by somatosensory system disease or damage), and mixed syndromes (coexistence of nociceptive and neuropathic pain). ${ }^{34}$ However, even the terminology of pain becomes challenging and contentious. ${ }^{35}$ For example, the International Association for the Study of Pain is currently attempting to distinguish between "nociception" (a sensory process) and "pain" (a subjective phenomenon). ${ }^{36}$

Multiple mechanisms contribute to painful syndromes, including nociception, peripheral sensitization, central sensitization, phenotypic switches, ectopic excitability, structural reorganization, and compromised inhibitory systems. ${ }^{37-41}$ Hypersensitivity causes a mild stimulus to provoke pain out of proportion to the stimulus. Hypersensitivity may be categorized academically as allodynia (pain response to nonnociceptive stimuli) or hyperalgesia (increased pain sensitivity in response to nociceptive stimuli), ${ }^{37}$ although these phenomena may be difficult to distinguish clinically.

The mechanisms may act in different ways. Nociception requires an intact central nervous system; changes in the central nervous system are evident in chronic pain patients. ${ }^{42}$ Primary afferent or sensory neurons play an important role in nociceptive pain processing, thus involving the peripheral 
nervous system. ${ }^{42}$ Inflammation, altered sympathetic and catecholaminergic function, changes in somatosensory processing in spinal cord and brain, pressure, temperature, neuropathic components, along with psychological factors, may also play a role in acute and chronic pain syndromes. ${ }^{43}$ The transition from acute to chronic pain is not thoroughly understood, but it is likely to involve the interaction among immune, endocrine, and nervous systems ${ }^{44}$ and, therefore, progressing central and peripheral sensitization. ${ }^{45}$ Other factors no doubt play a role. A study of trauma patients $(\mathrm{n}=290)$ identified as risk predictors for the transition to chronic pain - that is, pain that persists beyond 3 months: older age, female sex, past alcohol dependence, the amount of morphine equivalents administered on the day of assessment, and attitudes about pain control. ${ }^{46} \mathrm{~A}$ two-dimension positron emission tomography scan study of 20 cancer patients found preferential activation of the prefrontal cortex in patients with chronic pain but not in similar patients without pain. ${ }^{47}$ The prefrontal cortex is associated with emotional response, which may account for the emotional component of chronic pain.

In certain rheumatic pain conditions, selective serotonin reuptake inhibitors, serotonin and noradrenalin reuptake inhibitors, as well as tricyclic antidepressants have been shown to exert an analgesic effect that is distinct from their ability to treat depression, fatigue, and sleep disturbances. ${ }^{48,49}$ However, the evidence for the efficacy of these drugs in treating common pain syndromes (headache, low back pain, fibromyalgia, postherpetic neuralgia, and others) remains equivocal and, at times, conflicting. ${ }^{50,51}$ This suggests that these common pain syndromes may involve different pain mechanisms.

The accurate assessment of pain is challenging because pain perception is subjectively reported and may be influenced by the patient's attitude about health, disease, and personal expectations. ${ }^{52}$ These differences may be more than just idiosyncratic. For example, men and women not only experience pain differently, they may respond to analgesics differently. ${ }^{53}$

Pain may be a potentially serious comorbid condition, affecting medical and surgical outcomes. ${ }^{23}$ Maladaptive chronic pain may even be regarded as a disease in its own right. ${ }^{37}$ As such, it is crucial to devote our attention to better understanding and superior management of patients dealing with acute and chronic pain. The identification and increased understanding of the multiple mechanisms of pain has been a major advance.

\section{Commonly used agents in the treatment of pain}

Since the dawn of medicine, clinicians have treated pain (Table 1). As early as $3000 \mathrm{BC}$, natural salicylates were applied for the treatment of pain and Hippocrates reported on the analgesic efficacy of opium as early as 400 BC. However, in early medicine, these narcotics enjoyed a dubious reputation because of their potential for misuse, potentially life-threatening side effects, and withdrawal symptoms. ${ }^{54}$ Chemistry-based anti-inflammatory therapy began in 1897 with the discovery of aspirin, leading to advances in other pharmacological options, including NSAIDs. In 1986, the WHO proposed its well-known "pain ladder," which calls for the treatment of cancer pain based on level of pain intensity rather than the underlying mechanism, in that it advocates the use of nonopioid agents (such as aspirin, paracetamol, and NSAIDs) for mild pain, weak opioids for moderate pain (tramadol), and strong opioids (morphine) for severe pain. ${ }^{1}$ The multimechanistic nature of pain is recognized in the WHO ladder insofar as it includes adjuvant medications to treat pain.

When the WHO ladder was introduced in 1986 , oxycodone, hydromorphone, and buprenorphine did not exist. Tramadol was not available worldwide until the 1990s. Transdermal delivery systems for opioids were unknown in 1986. Methadone, not listed on the WHO pain ladder, existed in 1986, but its analgesic benefits in treating cancer pain were unknown. The first guidelines for neuropathic pain management were not published until the first decade of the 21 st century ${ }^{55-58}$ and the neuropathic treatment model differs from the WHO ladder (opioids are adjuvants in neuropathic pain management). Thus, in particular, the pain model should be updated with new pharmacological agents (new opioids, gabapentinoids, etc) according to new insights into adjuvant and multimodal therapies. ${ }^{59}$ It should also be noted that all treatment options may be combined with nonpharmacological approaches and patients may benefit from these multidisciplinary efforts.

\section{Weighing the risks of treatment with high-dose NSAIDs and paracetamol}

Paracetamol or acetaminophen is frequently grouped with NSAIDs, but it is actually an aniline analgesic. The terms "paracetamol" and "acetaminophen" reflect only geographical differences: "acetaminophen" is the term used in the USA, Canada, Hong Kong, Iran, and certain Latin American countries, such as Colombia, while "paracetamol" 
Table I Milestones in analgesic agents

\begin{tabular}{|c|c|}
\hline Year & Event \\
\hline $3000 \mathrm{BC}$ & First description of the use of myrtle leaves as systemic pain treatment \\
\hline Approximately $400 \mathrm{BC}$ & Hippocrates reports on the pain-relieving properties of opium in treating internal diseases and diseases of women \\
\hline 1527 & Paracelsus prescribes opium with other agents as an analgesic \\
\hline 1680 & $\begin{array}{l}\text { Thomas Sydenham introduces Sydenham's laudanum (opium mixed with wine and herbs), which becomes a } \\
\text { popular home remedy }\end{array}$ \\
\hline 1803 & Friedrich Sertürner discovers the active ingredient in opium - morphine \\
\hline 1827 & Merck and Company begin first commercial manufacture of morphine \\
\hline 1877 & $\begin{array}{l}\text { Synthesis of paracetamol (acetaminophen) at Johns Hopkins University is completed, but the drug would not be } \\
\text { used in patients for another } 10 \text { years }\end{array}$ \\
\hline 1890 & Morphine, legal in the USA, is taxed by Congress \\
\hline 1895 & $\begin{array}{l}\text { Bayer Company adds acetyls to morphine to reduce side effects to create a drug that would be marketed in } 1898 \\
\text { as Heroin (trade name) }\end{array}$ \\
\hline 1897 & $\begin{array}{l}\text { Discovery of aspirin, named for Spiraea (meadowsweet), one of many salicylate sources used to treat pain in the } \\
\text { nineteenth century }\end{array}$ \\
\hline 1905 & USA bans opium (but not opioid drugs) \\
\hline 1910 & $\begin{array}{l}\text { Heroin, marketed as a cough suppressant and morphine substitute, is taken off the market when it is found it is } \\
\text { more addictive than morphine }\end{array}$ \\
\hline 1914 & $\begin{array}{l}\text { The Harrison Narcotics Act in the USA requires physicians and pharmacists who prescribe or dispense narcotics } \\
\text { to register (and pay a tax) }\end{array}$ \\
\hline 1953 & Paracetamol (acetaminophen) first marketed in the USA by Sterling-Winthrop Company \\
\hline 1955 & McNeil Laboratories first markets Tylenol ${ }^{\circledR}$ brand (paracetamol) in the USA \\
\hline 1956 & Frederick Stearns and Company first markets Panadol in the UK \\
\hline 1963 & Development of nonsteroidal anti-inflammatory drugs (NSAIDs) \\
\hline 1971 & Understanding of the mechanism of action of aspirin \\
\hline$|990-199|$ & Discovery of cyclooxygenase-2 (COX-2) \\
\hline 1992 & COX-2 drug development \\
\hline $1998-1999$ & Celecoxib and rofecoxib introduced \\
\hline $2004-2006$ & Rofecoxib withdrawn from market \\
\hline 2005 & Warning of increased cardiovascular risk must be added to labeling for all NSAIDs in US (FDA requirement) \\
\hline $2006-2010$ & Warnings and dose restrictions on NSAIDs \\
\hline 2009 & Dextropropoxyphene withdrawn from market in the European Union \\
\hline 2010 & FDA launches Safe Use Initiative \\
\hline 2010 & Propoxyphene withdrawn from market in the USA \\
\hline
\end{tabular}

Abbreviation: FDA, US Food and Drug Administration.

is used in Europe, Africa, and most of Asia. The drug is sometimes abbreviated to "APAP" in all geographic regions. The mechanism of action of paracetamol is not well understood and several models have been proposed, all of which have certain strengths and limitations. ${ }^{60}$ Paracetamol is metabolized mainly by conjugation with sulfate and glucuronide, with about $5 \%$ to $10 \%$ of the drug oxidized by the cytochrome P450 metabolic pathway (mostly CYP2E1 and CYP3A4) to a toxic electrophilic metabolite, $N$-acetylp-benzoquinone imine (NAPQI). NAPQI is subsequently detoxified by glutathione and eliminated in the urine or bile. ${ }^{61}$ If any residual NAPQI is not detoxified in this manner, it may bind to hepatocytes, where it can lead to cellular necrosis. At appropriate doses in healthy individuals, the small amounts of NAPQI produced by paracetamol metabolism can be effectively eliminated with glutathione. However, at higher doses, paracetamol is associated with serious hepatic toxicity. ${ }^{62}$ In fact, paracetamol toxicity is the leading indication for liver transplantation in the $\mathrm{UK}^{63}$ and one of the most common causes of poisoning ${ }^{64}$ and acute liver failure ${ }^{65}$ in the USA. Paracetamol has also been linked to hypertension, ${ }^{66-68}$ which is probably caused by the considerable sodium content present in each paracetamol tablet. Thus, there are still unanswered questions about these side effects, including their extent. ${ }^{69-71}$

NSAIDs encompass a diverse group of drugs that reduce pronociceptive and proinflammatory prostaglandins and other chemical mediators by inhibiting their biotransformation in the arachidonic cascade, a reaction catalyzed by cyclooxygenase (COX) isoenzymes. ${ }^{72}$ In this way, they are similar to aspirin. ${ }^{73}$

The safety of many drugs, including pain drugs, has not been studied in as much detail as safety issues of NSAIDs and especially selective COX-2 inhibitors (coxibs). Nonselective NSAIDs block COX, namely COX-1 and 
COX-2, blocking the synthesis of prostaglandins and consequently shunting arachidonic acid into the lipoxygenase pathway, producing leukotrienes. Leukotrienes are powerful bronchoconstrictors and impair mucociliary clearance, resulting in increased mucus production, mucus filtration, and edema. Obviously, NSAID use has been associated with bronchospasm. ${ }^{74}$ Coxibs selectively block COX-2 and include such drugs as celecoxib, valdecoxib, and rofecoxib, limiting the COX-1-related inhibition to vital housekeeping functions. All NSAIDs are associated with dose-dependent toxicity, manifesting as gastrointestinal symptoms, including dyspepsia, ulceration, and bleeding, as well as cardio-renal complications including fluid retention, hypertension, and renal dysfunction. ${ }^{75-77}$ A recent study found even short-term use of NSAIDs was associated with increased risk of death in patients with a history of myocardial infarction (hazard ratio 1.45 ; 95\% confidence interval: $1.29-1.62){ }^{78}$

For such reasons, NSAIDs, including coxibs, should not be prescribed as a panacea for all pains, but restricted to pain related to tissue damage and/or inflammation, in accordance to their mechanism of action. ${ }^{79-81}$ NSAIDs are to be used cautiously, in patients with or at elevated risk for cardiovascular disease $\mathrm{e}^{29,78,79,81-84}$ or gastrointestinal complications. ${ }^{79,81,85}$

\section{Pharmacological aspects: why combinations might be better than single agents}

Rarely does a single known mechanism cause pain. Obviously, no single analgesic agent can fully address multiple mechanisms of pain. Combination analgesic products have been effective because they activate multiple pain-inhibitory pathways and offer a broader spectrum of relief. ${ }^{86}$ This may include multiple afferents and pathways as well as multiple processes.

Combination analgesics might reduce adverse events. ${ }^{86}$ A given analgesic provides pain relief at a specific dosage and is associated with dose-dependent adverse effects. Combining analgesics may allow for lower doses of the individual agents, with doses possibly low enough to significantly reduce potential adverse events. While the theory of combination analgesic products holds promise, combination products require rigorous scrutiny and testing since not all combinations are ideal.

Combining two or more agents may result in an additive or synergistic analgesic effect. ${ }^{86,87}$ When agents are combined, the combination effect may be greater than, less than, or the same as the predicted magnitude of effect, resulting in synergistic, sub-additive, or additive effects, respectively. Such effects are calculated mathematically based on the concept of dose equivalence, defined as doses of each drug that yield the same magnitude of effect when each is used by itself. These calculations compare actual versus expected effects in graphic representations of dose combinations known as isoboles ${ }^{88-92}$ (Figure 1). Isobolographic analysis is well accepted and has been used with many drug combinations. ${ }^{93,94}$ Drugs with a constant potency ratio have linear isoboles of additivity, ${ }^{93-95}$ but drugs with variable potency ratios can be analyzed as well. ${ }^{96}$ Receptor saturation of the agents can also be assessed. ${ }^{97}$

Combination analgesic products are common and include, but are not limited to, such products as Empirin ${ }^{\circledR}$ (paracetamol + codeine), Vicodin ${ }^{\circledR}$ (paracetamol + hydrocodone), Percocet ${ }^{\circledR}$ (paracetamol + oxycodone), and Zaldiar ${ }^{\circledR}$ or Ultracet ${ }^{\circledR}$ (paracetamol + tramadol). Table 2 lists selected studies of fixed-dose combinations with paracetamol, all of them having demonstrated good efficacy in several chronic pain conditions.

As an example of fixed-dose combination, the participants of the meeting discussed tramadol/paracetamol because this product has been more extensively evaluated than other combination products. The theoretical rationale for the combination agents described needs to be backed by clinical evidence because, in some cases, additive benefits do not result in clinically meaningful differences. Tramadol/Paracetamol is - to our knowledge the only fixed-dose combination where both the dual mechanism of action of tramadol and the analgesic synergy between the two compounds have been demonstrated in both preclinical studies (mouse model) and human companion studies using essentially the same study design. ${ }^{30-33}$ Table 3 provides an overview of the relevant results. Further study of tramadol/paracetamol combination analgesia in chronic pain syndromes is warranted to better evaluate long-term safety and efficacy.

According to these and later studies, the mechanisms of action of tramadol may be described, respectively as: a weak agonist effect at the $\mu$-opioid receptors, inhibition of serotonin reuptake, and inhibition of norepinephrine reuptake. ${ }^{98}$

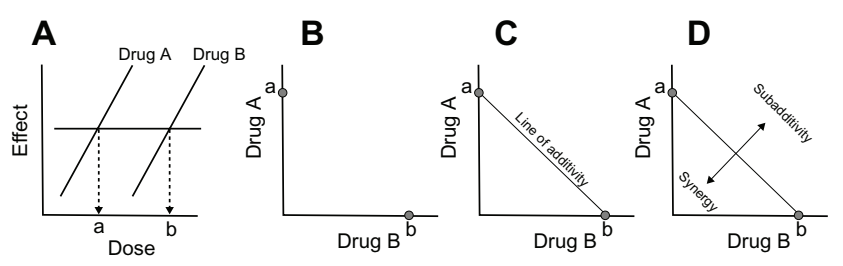

Figure I Representation of isobolographic analysis. Equi-effective doses of two drugs are determined $(\mathbf{A})$ and graphed on Cartesian coordinates (B). The predicted effect of various ratios of combinations of these drugs is simple additivity (C). Actual results on, above, or below the predicted line of additivity (D) are indicative of additive, sub-additive, or supra-additive (synergistic) interaction, respectively. 
Table 2 Selected clinical studies using fixed-dose combination products with paracetamol

\begin{tabular}{|c|c|c|c|c|}
\hline Study & $\mathbf{N}$ & Agents & Results & Comments \\
\hline \multicolumn{5}{|l|}{ Postoperative pain } \\
\hline \multicolumn{5}{|l|}{ Dental } \\
\hline $\begin{array}{l}\text { Fricke et al }{ }^{139} \\
\text { Double-blind, } \\
\text { randomized }\end{array}$ & 200 & $\begin{array}{l}\text { - Tramadol/APAP } 37.5 \mathrm{mg} / 325 \mathrm{mg} \\
\text { - Tramadol/APAP } 75 \mathrm{mg} / 650 \mathrm{mg} \\
\text { - Hydrocodone/APAP } 10 \mathrm{mg} / 650 \mathrm{mg} \\
\text { - Placebo } \\
\text { - Single dose }\end{array}$ & $\begin{array}{l}\text { - Comparable analgesia between tramadol/ } \\
\text { ATAP } 75 \mathrm{mg} / 650 \mathrm{mg} \text { and hydrocodone/ } \\
\text { APAP but better tolerability for } \\
\text { tramadol/ATAP } \\
\text { - Nausea and vomiting were } 50 \% \text { lower } \\
\text { with tramadol/APAP } 75 \mathrm{mg} / 650 \mathrm{mg} \text { than } \\
\text { with hydrocodone/APAP }\end{array}$ & $\begin{array}{l}\text { Removal of } \geq 2 \text { impacted } \\
\text { third molars }\end{array}$ \\
\hline $\begin{array}{l}\text { MacLeod et al'140 } \\
\text { Double-blind, } \\
\text { randomized, } \\
\text { parallel-group }\end{array}$ & 82 & $\begin{array}{l}\text { - Codeine/APAP } 30 \mathrm{mg} / 1000 \mathrm{mg} \\
\text { - APAP } 1000 \mathrm{mg} \\
\text { - } 3 \text { doses over } 8 \text { hours }\end{array}$ & $\begin{array}{l}\text { - Combination significantly more effective } \\
\text { in pain control } \\
\text { - Similar AE incidences }\end{array}$ & $\begin{array}{l}\text { Removal of impacted third } \\
\text { molars }\end{array}$ \\
\hline $\begin{array}{l}\text { Edwards et al }{ }^{141} \\
\text { Meta-analysis, } \\
\text { randomized studies }\end{array}$ & $\begin{array}{l}5 \\
\text { studies }\end{array}$ & $\begin{array}{l}\text { - Tramadol/APAP } 75 \mathrm{mg} / 650 \mathrm{mg} \\
\text { or II } 2.5 \mathrm{mg} / 975 \mathrm{mg} \\
\text { - Tramadol } 75 \mathrm{mg} \\
\text { - Single dose }\end{array}$ & $\begin{array}{l}\text { - NNT for at least } 50 \% \text { pain relief over } \\
\text { 6-hour period: } \\
-2.6 \text { tramadol/APAP } \\
-9.9 \text { tramadol }\end{array}$ & $\begin{array}{l}\text { NNH was } 5.4(4.0-8.2) \\
\text { for tramadol/APAP and } \\
5.0(3.7-7.3) \text { for tramadol }\end{array}$ \\
\hline $\begin{array}{l}\text { Jung et al }{ }^{142} \\
\text { Randomized }\end{array}$ & 128 & $\begin{array}{l}\text { - Tramadol/APAP } 75 \mathrm{mg} / 650 \mathrm{mg} \\
\text { - Codeine/APAP/ibuprofen } \\
20 \mathrm{mg} / 500 \mathrm{mg} / 400 \mathrm{mg} \\
\text { - Single dose }\end{array}$ & $\begin{array}{l}\text { - Comparable onset of analgesia, analgesic } \\
\text { efficacy, and safety profile }\end{array}$ & $\begin{array}{l}\text { Extraction of } \geq 1 \\
\text { impacted third molar } \\
\text { requiring bone removal }\end{array}$ \\
\hline $\begin{array}{l}\text { Litkowski et al }{ }^{143} \\
\text { Double-blind, } \\
\text { randomized, } \\
\text { placebo-controlled, } \\
\text { parallel-group }\end{array}$ & 249 & $\begin{array}{l}\text { - Oxycodone/ibuprofen } 5 \mathrm{mg} / 400 \mathrm{mg} \\
\text { - Oxycodone/APAP } 5 \mathrm{mg} / 325 \mathrm{mg} \\
\text { - Hydrocodone/APAP } 7.5 \mathrm{mg} / 500 \mathrm{mg} \\
\text { - Placebo } \\
\text { - Single dose }\end{array}$ & $\begin{array}{l}\text { Oxycodone/lbuprofen with significantly } \\
\text { better pain relief than other treatments } \\
\text { AE rate of oxycodone/ibuprofen similar } \\
\text { to placebo and } 2 \text {-fold lower to other } \\
2 \text { active agents }\end{array}$ & $\begin{array}{l}\text { Removal of } 2 \text { or more } \\
\text { impacted third molars }\end{array}$ \\
\hline $\begin{array}{l}\text { Daniels et al }{ }^{144} \\
\text { Double-blind, } \\
\text { randomized, } \\
\text { parallel-group, } \\
\text { placebo-controlled }\end{array}$ & 678 & $\begin{array}{l}\text { - Ibuprofen/APAP } 400 \mathrm{mg} / 1000 \mathrm{mg} \\
\text { - Ibuprofen/APAP } 200 \mathrm{mg} / 500 \mathrm{mg} \\
\text { - Ibuprofen/codeine } 400 \mathrm{mg} / 25.6 \mathrm{mg} \\
\text { - Codeine/APAP } 30 \mathrm{mg} / 1000 \mathrm{mg} \\
\text { - Placebo } \\
\text { - Single dose }\end{array}$ & $\begin{array}{l}\text { - Both doses of ibuprofen/APAP with } \\
\text { significantly more effective pain relief } \\
\text { than placebo and codeine/APAP } \\
\text { - Ibuprofen/APAP } 400 \mathrm{mg} / 1000 \mathrm{mg} \\
\text { significantly superior to ibuprofen/ } \\
\text { codeine; ibuprofen/APAP } 200 \mathrm{mg} / 500 \mathrm{mg} \\
\text { noninferior to ibuprofen/codeine } \\
\text { - AE rates were higher for codeine } \\
\text { combinations }\end{array}$ & $\begin{array}{l}\text { Removal of } \geq 3 \text { impacted } \\
\text { third molars }\end{array}$ \\
\hline \multicolumn{5}{|l|}{ Other procedures } \\
\hline $\begin{array}{l}\text { White et al }{ }^{145} \\
\text { Double-blind, } \\
\text { randomized, } \\
\text { parallel-group }\end{array}$ & 252 & $\begin{array}{l}\text { - Hydrocodone/APAP } \\
7.5 \mathrm{mg} / 750 \mathrm{mg} \\
\text { - Ketorolac } 10 \mathrm{mg} \\
\text { - Placebo } \\
\text { - Every } 6 \text { hours for up to } 3 \text { days }\end{array}$ & $\begin{array}{l}\text { - No difference in pain relief between the } \\
\text { active agents after arthroscopic } \\
\text { procedures, both superior to placebo } \\
\text { - No difference in pain relief between all } \\
3 \text { groups for laparoscopic procedures } \\
\text { - AE incidences similar for both active } \\
\text { agents, except higher incidence of } \\
\text { postoperative dizziness for } \\
\text { hydrocodone/APAP }\end{array}$ & $\begin{array}{l}\text { Ambulatory arthroscopic } \\
\text { or laparoscopic tubal } \\
\text { ligation }\end{array}$ \\
\hline $\begin{array}{l}\text { Palangio et al }{ }^{46} \\
\text { Double-blind, } \\
\text { randomized, } \\
\text { placebo-controlled }\end{array}$ & 180 & $\begin{array}{l}\text { - Hydrocodone/ibuprofen } \\
\text { I5 mg/400 mg } \\
\text { - Oxycodone/APAP } 10 \mathrm{mg} / 650 \mathrm{mg} \\
\text { - Placebo } \\
\text { - Single dose }\end{array}$ & $\begin{array}{l}\text { Both active combinations provided } \\
\text { significantly better pain relief than } \\
\text { placebo; hydrocodone/ibuprofen superior } \\
\text { to oxycodone/APAP at some time points } \\
\text { - AEs similar for active agents }\end{array}$ & $\begin{array}{l}\text { Obstetric or gynecological } \\
\text { surgery }\end{array}$ \\
\hline $\begin{array}{l}\text { Smith et al }{ }^{132} \\
\text { Double-blind, } \\
\text { randomized, } \\
\text { placebo-controlled }\end{array}$ & 305 & $\begin{array}{l}\text { - Tramadol/APAP } 75 \mathrm{mg} / 650 \mathrm{mg} \\
\text { - Codeine/APAP } 60 \mathrm{mg} / 600 \mathrm{mg} \\
\text { - Placebo } \\
\text { - Mean daily dose: } \\
\text { - tramadol/APAP } 163 \mathrm{mg} / 1415 \mathrm{mg} \\
\text { - codeine/APAP } 130 \mathrm{mg} / 1296 \mathrm{mg}\end{array}$ & $\begin{array}{l}\text { - Both active combinations provided } \\
\text { significantly greater pain relief than } \\
\text { placebo; scores were similar for } \\
\text { tramadol/APAP and codeine/APAP } \\
\text { - Tramadol/APAP was better tolerated } \\
\text { than codeine/APAP but AE rates were } \\
\text { similar for both active groups }\end{array}$ & $\begin{array}{l}\text { Orthopedic and } \\
\text { abdominal surgery }\end{array}$ \\
\hline
\end{tabular}


Table 2 (Continued)

\begin{tabular}{|c|c|c|c|c|}
\hline Study & $\mathbf{N}$ & Agents & Results & Comments \\
\hline $\begin{array}{l}\text { Sniezek et al }{ }^{147} \\
\text { Double-blind, } \\
\text { randomized }\end{array}$ & 210 & $\begin{array}{l}\text { - APAP } 1000 \text { mg } \\
\text { - Ibuprofen/APAP } 400 \text { mg/l000 mg } \\
\text { - Codeine/APAP } 30 \text { mg/ } 325 \text { mg } \\
\text { - Immediately after surgery and } \\
\text { every } 4 \text { hours for up to } 4 \text { doses }\end{array}$ & $\begin{array}{l}\text { - Ibuprofen/APAP superior to other } \\
2 \text { treatments in pain control } \\
\text { - Higher rate of AEs under codeine/APAP } \\
\text { compared with ibuprofen/APAP and } \\
\text { APAP alone }\end{array}$ & $\begin{array}{l}\text { Mohs micrographic } \\
\text { surgery and } \\
\text { reconstruction for head } \\
\text { and neck skin cancer }\end{array}$ \\
\hline $\begin{array}{l}\text { Rawal et al } \\
\text { Randomized, } \\
\text { double-blind, } \\
\text { double-dummy, } \\
\text { parallel-group }\end{array}$ & 261 & $\begin{array}{l}\text { - Tramadol/APAP } 37.5 \text { mg/325 mg } \\
\text { - Tramadol } 50 \text { mg } \\
\text { - Before and immediately after } \\
\text { surgery and every } 6 \text { hours } \\
\text { thereafter }\end{array}$ & $\begin{array}{l}\text { - Comparable analgesic efficacy, fewer AEs } \\
\text { with tramadol/APAP compared } \\
\text { with tramadol monotherapy } \\
\text { - Tramadol/APAP reduced tramadol } \\
\text { consumption by } 24 \%\end{array}$ & $\begin{array}{l}\text { Ambulatory hand surgery } \\
\text { with iv regional anesthesia }\end{array}$ \\
\hline \multicolumn{5}{|l|}{ Musculoskeletal pain } \\
\hline $\begin{array}{l}\text { Mullican and } \\
\text { Lacy } \\
\text { Double-blind, } \\
\text { randomized }\end{array}$ & 462 & $\begin{array}{l}\text { - Tramadol/APAP } 37.5 \text { mg/325 mg } \\
\text { - Codeine/APAP } 30 \text { mg/300 mg } \\
\text { - Mean daily dose: } \\
\text { - Tramadol/APAP } 131 \mathrm{mg} / \mathrm{l} / 33 \mathrm{mg} \\
\text { - Codeine/APAP } 105 \mathrm{mg} / \mathrm{l} 054 \mathrm{mg}\end{array}$ & $\begin{array}{l}\text { - Comparable efficacy, better tolerability } \\
\text { for tramadol/APAP }\end{array}$ & $\begin{array}{l}\text { Chronic, nonmalignant } \\
\text { low back pain and } \\
\text { osteoarthritis pain }\end{array}$ \\
\hline $\begin{array}{l}\text { Serrie et al }{ }^{149} \\
\text { Observational, } \\
\text { prospective, } \\
\text { open-label, in clinical } \\
\text { practice (ELZA), } \\
\text { mean therapy } \\
\text { duration } 16.6 \text { days }\end{array}$ & 5495 & $\begin{array}{l}\text { - Tramadol/APAP } 37.5 \text { mg/325 mg } \\
\text { - Mean daily dose } 139 \text { mg/l } 203 \text { mg }\end{array}$ & $\begin{array}{l}\text { - Significant reduction from baseline in } \\
\text { mean pain intensity score } \\
\text { - } 4.2 \% \text { of patients with AEs }\end{array}$ & $\begin{array}{l}\text { Majority of patients had } \\
\text { musculoskeletal pain }\end{array}$ \\
\hline $\begin{array}{l}\text { Mejjad et al }{ }^{150} \\
\text { Observational, } \\
\text { prospective, } \\
\text { open-label, in clinical } \\
\text { practice (SALZA), } \\
\text { median treatment } \\
30 \text { days }\end{array}$ & 2663 & $\begin{array}{l}\text { - Tramadol/APAP } 37.5 \text { mg/325 mg } \\
\text { - Mean daily dose } 143 \text { mg/l } 235 \text { mg }\end{array}$ & $\begin{array}{l}\text { - Marked reduction from baseline in mean } \\
\text { pain intensity score (from } 6.1 \pm 1.6 \text { at } \\
\text { baseline to } 3.0 \pm 1.8 \text { at final assessment) } \\
\text { - } 91 \% \text { of patients were satisfied or } \\
\text { completely satisfied } \\
\text { - Rate of AEs was } 4.5 \%\end{array}$ & $\begin{array}{l}\text { Patients aged } \geq \\
65 \text { years, primarily with } \\
\text { musculoskeletal pain }\end{array}$ \\
\hline \multicolumn{5}{|l|}{ Osteoarthritis } \\
\hline $\begin{array}{l}\text { Emkey et al }{ }^{151} \\
\text { Double-blind, } \\
\text { randomized, } \\
\text { placebo-controlled }\end{array}$ & 306 & $\begin{array}{l}\text { - Tramadol/APAP } 37.5 \text { mg/325 mg } \\
\text { - Placebo } \\
\text { - Mean daily dose } 154 \text { mg// } 332 \text { mg }\end{array}$ & $\begin{array}{l}\text { - Significant pain relief, significant } \\
\text { improvement in medical assessments, } \\
\text { physical function, and subject's and } \\
\text { investigator's overall assessment } \\
\text { - I3\% of tramadol/APAP and } 4 \% \text { of placebo } \\
\text { patients discontinued owing to AEs }\end{array}$ & $\begin{array}{l}\text { Add-on for patients with } \\
\text { inadequate pain control } \\
\text { by celecoxib or rofecoxib }\end{array}$ \\
\hline $\begin{array}{l}\text { Corsinovi et al } \\
\text { Randomized, } \\
\text { single-blind }\end{array}$ & 154 & $\begin{array}{l}\text { - Average dose at end of study: } \\
\text { - Oxycodone/APAP } 16 \mathrm{mg} / 900 \mathrm{mg} \\
\text { - Codeine/APAP II5 mg/1916 mg } \\
\text { - Conventional therapy (NSAIDs, } \\
\text { APAP, COX-2 inhibitors) }\end{array}$ & $\begin{array}{l}\text { - Significantly greater pain reductions for } \\
\text { oxycodone/APAP and codeine/APAP } \\
\text { compared with conventional therapy } \\
\text { - AE rates did not differ between groups }\end{array}$ & Elderly females \\
\hline $\begin{array}{l}\text { Pareek et al }{ }^{153} \\
\text { Randomized, } \\
\text { open-label }\end{array}$ & 199 & $\begin{array}{l}\text { - Aceclofenac/APAP } \\
100 \mathrm{mg} / 500 \mathrm{mg} \text { bid } \\
\text { - Aceclofenac } 100 \mathrm{mg} \text { bid }\end{array}$ & $\begin{array}{l}\text { - Combination superior in pain intensity } \\
\text { differences, sum of pain intensity } \\
\text { differences, peak pain intensity } \\
\text { differences and patients'/ } \\
\text { investigators' assessments } \\
\text { - Combination had more rapid onset } \\
\text { of action } \\
\text { - AE rate similar in both groups }\end{array}$ & Knee flare-up \\
\hline $\begin{array}{l}\text { Pareek et al }{ }^{154} \\
\text { Randomized, } \\
\text { double-blind }\end{array}$ & 220 & $\begin{array}{l}\text { - Etodolac/APAP } \\
300 \mathrm{mg} / 500 \mathrm{mg} \text { bid } \\
\text { - Etodolac } 300 \mathrm{mg} \text { bid }\end{array}$ & $\begin{array}{l}\text { - Compared with etodolac monotherapy, } \\
\text { etodolac/APAP was superior in reducing } \\
\text { pain intensity and improvement of } \\
\text { function } \\
\text { - Results noticeable within } 30 \text { minutes } \\
\text { of first dose } \\
\text { - Similar AE rates for both groups }\end{array}$ & Knee flare-up \\
\hline
\end{tabular}


Table 2 (Continued)

\begin{tabular}{|c|c|c|c|c|}
\hline Study & $\mathbf{N}$ & Agents & Results & Comments \\
\hline $\begin{array}{l}\text { Doherty et al }{ }^{155} \\
\text { Double-blind, } \\
\text { randomized, } \\
\text { parallel-group }\end{array}$ & 892 & $\begin{array}{l}\text { - Ibuprofen } 400 \text { mg tid } \\
\text { - APAP } 1000 \text { mg tid } \\
\text { - Ibuprofen/APAP } 200 \text { mg/500 mg tid } \\
\text { - Ibuprofen/APAP } 400 \text { mg//000 mg tid }\end{array}$ & $\begin{array}{l}\text { - Ibuprofen/APAP, at nonprescription } \\
\text { doses, confers modest short-term } \\
\text { benefits } \\
\text { - Decreases in hemoglobin by } \geq \mathrm{I} g / \mathrm{dL} \\
\text { occurred in all groups but were twice as } \\
\text { frequent in patients taking } 2 \text { combination } \\
\text { tablets daily compared with monotherapy }\end{array}$ & $\begin{array}{l}\geq 40 \text { years of age } \\
\text { Chronic knee pain, } \\
85 \% \text { osteoarthritis }\end{array}$ \\
\hline $\begin{array}{l}\text { Conaghan et al }{ }^{156} \\
\text { Open-label, } \\
\text { randomized, } \\
\text { parallel-group }\end{array}$ & 220 & $\begin{array}{l}\text { - 7-day buprenorphine patches } \\
\text { (range } 5-25 \mu \mathrm{g} / \mathrm{hour} \text { ) + } \\
\text { APAP } 1000 \mathrm{mg} \text { qid } \\
\text { - Codeine/APAP range } 16-60 \mathrm{mg} / \\
1000 \mathrm{mg} \text { qid }\end{array}$ & $\begin{array}{l}\text { - Noninferiority of patch + APAP to } \\
\text { codeine/APAP combination regarding } \\
\text { analgesic efficacy } \\
\text { - Comparable incidence of AEs } \\
\text { - High withdrawal rates in both groups }\end{array}$ & $\begin{array}{l}\text { - Hip and/or knee pain } \\
\text { - } \geq 60 \text { years of age }\end{array}$ \\
\hline \multicolumn{5}{|l|}{ Low back pain } \\
\hline $\begin{array}{l}\text { Palangio et al }{ }^{157} \\
\text { Double-blind, } \\
\text { randomized, } \\
\text { parallel-group }\end{array}$ & 147 & $\begin{array}{l}\text { - Hydrocodone/ibuprofen } \\
7.5 \mathrm{mg} / 200 \mathrm{mg} \\
\text { - Oxycodone/APAP } 5 \mathrm{mg} / 325 \mathrm{mg} \\
\text { - Mean daily dose: } \\
\text { - Hydrocodone/ibuprofen } \\
\text { I3.5 mg/360 mg } \\
\text { - Oxycodone/APAP II mg/7I5 mg }\end{array}$ & $\begin{array}{l}\text { - No significant differences between the } \\
\text { groups in efficacy and AEs }\end{array}$ & Acute pain \\
\hline $\begin{array}{l}\text { Ruoff et al }{ }^{158} \\
\text { Double-blind, } \\
\text { randomized }\end{array}$ & 318 & $\begin{array}{l}\text { - Tramadol/APAP } 37.5 \text { mg/325 mg } \\
\text { - Placebo } \\
\text { - Mean daily dose } 158 \text { mg/l } 365 \text { mg }\end{array}$ & $\begin{array}{l}\text { - Significantly improved outcome in all } \\
\text { efficacy measures compared with placebo } \\
\text { - Discontinuation due to AEs was } 19 \% \text { for } \\
\text { combination and } 6 \% \text { for placebo }\end{array}$ & Chronic pain \\
\hline $\begin{array}{l}\text { Perrot et al }{ }^{159} \\
\text { Double-blind, } \\
\text { randomized, } \\
\text { parallel-group }\end{array}$ & 119 & $\begin{array}{l}\text { - Tramadol/APAP } 37.5 \text { mg/325 mg } \\
\text { - Tramadol } 50 \text { mg } \\
\text { - Mean daily dose: } \\
\text { - Tramadol/APAP } 172 \text { mg/l495 mg } \\
\text { - Tramadol } 227 \mathrm{mg}\end{array}$ & $\begin{array}{l}\text { - Comparable analgesic efficacy with } \\
\text { significantly fewer AEs with tramadol/ } \\
\text { APAP } \\
\text { - Tramadol/APAP reduced tramadol } \\
\text { consumption by } 24 \%\end{array}$ & Subacute pain \\
\hline $\begin{array}{l}\text { Fibromyalgia } \\
\text { Bennett et al } \\
\text { Double-blind, } \\
\text { randomized, } \\
\text { placebo-controlled }\end{array}$ & 315 & $\begin{array}{l}\text { - Tramadol/APAP } 37.5 \text { mg/325 mg } \\
\text { - Placebo } \\
\text { - Mean daily dose } 150 \text { mg// } 300 \text { mg }\end{array}$ & $\begin{array}{l}\text { - Significantly better pain relief and health- } \\
\text { related QoL with combination therapy } \\
\text { - Discontinuation due to AEs was } 19 \% \text { for } \\
\text { combination and } 12 \% \text { for placebo }\end{array}$ & \\
\hline \multicolumn{5}{|c|}{ Rheumatoid arthritis } \\
\hline $\begin{array}{l}\text { Lee et } \text { al }^{161} \\
\text { Double-blind, } \\
\text { randomized, } \\
\text { placebo-controlled }\end{array}$ & 277 & $\begin{array}{l}\text { - Tramadol/APAP } \\
37.5 \mathrm{mg} / 325 \mathrm{mg} \text { tid } \\
\text { - Placebo }\end{array}$ & $\begin{array}{l}\text { - Significant improvement in pain relief, } \\
\text { significant reduction in pain intensity, } \\
\text { no difference in physical function, } \\
\text { significantly higher rate of AEs } \\
\text { - Discontinuation due to AEs was } 19 \% \text { for } \\
\text { combination and } 3 \% \text { for placebo }\end{array}$ & $\begin{array}{l}\text { Add-on for patients with } \\
\text { inadequate pain control } \\
\text { by conventional NSAIDs } \\
\text { and DMARDs }\end{array}$ \\
\hline $\begin{array}{l}\text { Raffaeli et al }{ }^{162} \\
\text { Open-label, } \\
\text { case series }\end{array}$ & 29 & $\begin{array}{l}\text { - Oxycodone/APAP } 5 \mathrm{mg} / 325 \mathrm{mg} \\
\text { - Mean daily dose at end of study } \\
\text { - } 14 \mathrm{mg} / 720 \mathrm{mg}\end{array}$ & $\begin{array}{l}\text { - } 42 \% \text { had good clinical response (EULAR) } \\
\text { and } 50 \% \text { showed } 20 \% \text { improvement } \\
\text { - No serious AEs }\end{array}$ & $\begin{array}{l}\text { Patients under } \\
\text { rheumatoid arthritis } \\
\text { therapy with biological } \\
\text { drugs were excluded }\end{array}$ \\
\hline \multicolumn{5}{|c|}{ Painful diabetic neuropathy } \\
\hline $\begin{array}{l}\text { Freeman et al }{ }^{130} \\
\text { Double-blind, } \\
\text { randomized, } \\
\text { placebo-controlled, } \\
\text { parallel-group }\end{array}$ & 313 & $\begin{array}{l}\text { - Tramadol/APAP } 37.5 \text { mg/325 mg } \\
\text { - Placebo } \\
\text { - Mean daily dose } 158 \text { mg/l } 365 \text { mg }\end{array}$ & $\begin{array}{l}\text { - Significantly greater improvements for all } \\
\text { measures of pain intensity, sleep } \\
\text { interference, and global impression as } \\
\text { well as several QoL measures and mood } \\
\text { - AE rate was } 60 \% \text { for the combination } \\
\text { and } 59 \% \text { for placebo, nausea, dizziness, } \\
\text { and somnolence significantly more } \\
\text { common under combination } \\
\text { - Discontinuation due to AEs was } 8 \% \text { for } \\
\text { combination and } 6.5 \% \text { for placebo }\end{array}$ & \\
\hline
\end{tabular}


Table 2 (Continued)

\begin{tabular}{|c|c|c|c|c|}
\hline Study & $\mathbf{N}$ & Agents & Results & Comments \\
\hline Ko et al ${ }^{129}$ & 163 & - Tramadol/APAP $37.5 \mathrm{mg} / 325 \mathrm{mg}$ & - Comparable mean reductions in pain & Patients with type 2 \\
\hline Open-label, & & - Gabapentin 300 mg & intensity and mean pain relief scores & diabetes aged $25-75$ years \\
\hline randomized & & $\begin{array}{l}\text { - Mean dose at final visit: } \\
\text { - Tramadol/APAP I58 mg//37I mg } \\
\text { - Gabapentin I575 mg }\end{array}$ & $\begin{array}{l}\text { - Comparable improvements in QoL } \\
\text { - Similar rates of AEs and discontinuation } \\
\text { due to AEs for both groups }\end{array}$ & $\begin{array}{l}\text { Dose adjusted to effect, } \\
\text { no rescue medication } \\
\text { during maintenance phase }\end{array}$ \\
\hline
\end{tabular}

Abbreviations: AEs, adverse events; APAP, paracetamol (acetaminophen); bid, twice daily; DMARD, disease-modifying antirheumatic drug; iv, intravenous; NSAIDs, nonsteroidal anti-inflammatory drugs; NNH, number needed to harm; NNT, number needed to treat; qid, four times per day; QoL, quality of life; tid, three times per day.

Table 3 Companion studies demonstrating mode of action of tramadol/paracetamol fixed-dose combination

\begin{tabular}{|c|c|c|c|c|}
\hline & \multicolumn{2}{|c|}{ Dual mechanism of action of tramadol } & \multicolumn{2}{|c|}{ Analgesic synergy between tramadol and paracetamol } \\
\hline & Mouse and rat model ${ }^{30}$ & Healthy male volunteers ${ }^{32}$ & Mouse model $\left.\right|^{31}$ & Healthy volunteers ${ }^{33}$ \\
\hline Design & & $\begin{array}{l}\text { Double-blind, randomized, } \\
\text { placebo-controlled, crossover }\end{array}$ & & $\begin{array}{l}\text { Double-blind, randomized, } \\
\text { placebo-controlled, crossover }\end{array}$ \\
\hline Agents & Tramadol iv & $\begin{array}{l}\text { - Tramadol } 100 \mathrm{mg} \text { oral dose } \\
\text { - } 3 \text { hours later, either placebo } \\
\text { injection or yohimbine iv } \\
0.1 \mathrm{mg} \mathrm{kg}^{-1}+\text { placebo or } \\
\text { yohimbine + naloxone } \\
\text { ( } \mu \text { opioid antagonist) } \\
0.8 \mathrm{mg} \text { iv }\end{array}$ & $\begin{array}{l}\text { Oral: } \\
\text { - APAP } \\
\text { - Tramadol } \\
\text { - Tramadol/APAP using } \\
\text { different fixed dose ratios } \\
\text { (TRAM/APAP ratios } \\
\text { tested were: 1000:1, } \\
\text { I00:1, 20:1, 3:1, I:I, } \\
\text { I:3, I:5, I:5.7, I:19, } \\
\text { I:50, I:100: 1:200, } \\
\text { I:800, and } 1: 1600 \text { ) }\end{array}$ & $\begin{array}{l}\text { iv infusions: } \\
\text { - APAP } 650 \mathrm{mg} \\
\text { - Tramadol } 75 \mathrm{mg} \\
\text { - Tramadol/APAP } 37.5 \mathrm{mg} / 325 \mathrm{mg} \\
\text { - Placebo }\end{array}$ \\
\hline Methods & $\begin{array}{l}\text { - Mouse acetylcholine-induced } \\
\text { abdominal constriction test } \\
\text { - Rat air-induced abdominal } \\
\text { constriction test } \\
\text { - Mouse/rat hotplate and } \\
\text { tail-flick tests } \\
\text { - Yohimbine ( } \alpha_{2} \text {-adrenoceptor } \\
\text { antagonist) and ritanserin } \\
\text { (5HT2A/2C antagonist) } \\
\text { antagonism in rats and mice }\end{array}$ & $\begin{array}{l}\text { - Induction of pain by } \\
\text { electrical stimulus } \\
\text { - Assessment of subjective } \\
\text { pain threshold (pain intensity } \\
\text { rating) and objective pain } \\
\text { threshold (R III nociceptive } \\
\text { reflex) for } 8 \text { hours after } \\
\text { tramadol intake }\end{array}$ & $\begin{array}{l}\text { - Acetylcholine bromide } \\
\text { injection } 30 \text { minutes } \\
\text { after analgesia delivery } \\
\text { - Assessment: occurrence } \\
\text { of a single abdominal } \\
\text { constriction response } \\
\text { - Estimation of } \mathrm{ED}_{50} \text { from } \\
\text { individual dose- } \\
\text { response curves }\end{array}$ & $\begin{array}{l}\text { - Induction of acute pain and } \\
\text { mechanical hyperalgesia by tran- } \\
\text { scutaneous electrical stimula- } \\
\text { tion at high current densities } \\
\text { - Drugs were delivered in a } \\
\text { I5-minute infusion starting } \\
30 \text { minutes after onset of } \\
\text { electrical stimulation } \\
\text { - Assessments before, during, } \\
\text { and I50 minutes after infusion }\end{array}$ \\
\hline Results & $\begin{array}{l}\text { - Tramadol produced } \\
\text { dose-related anti-nociception } \\
\text { in all tests } \\
\text { - This anti-nociceptive activity } \\
\text { was completely antagonized } \\
\text { by naloxone } \\
\text { - Administration of yohimbine } \\
\text { or ritanserin blocked anti- } \\
\text { nociceptive activity produced } \\
\text { by tramadol but not the one } \\
\text { produced by morphine }\end{array}$ & $\begin{array}{l}\text { - Tramadol induced a } \\
\text { significant increase in } \\
\text { both thresholds } \\
\text { - Yohimbine almost totally } \\
\text { reversed the subjective (67\%) } \\
\text { and objective ( } 97 \%) \\
\text { anti-nociceptive effect of } \\
\text { tramadol for } 2.8 \text { hours } \\
\text { - Addition of naloxone } \\
\text { abolished tramadol effects } \\
\text { (79\% for subjective, } 90 \% \text { for } \\
\text { objective pain threshold) }\end{array}$ & $\begin{array}{l}E D_{50} \text { values: } \\
\text { - Tramadol } 5.5 \pm 0.4 \\
\text { - APAP } 164.9 \pm 24.5\end{array}$ & $\begin{array}{l}\text { Pain reduction } \\
\text { (correction for placebo effects) } \\
\text { - Tramadol II.7\% } \pm 4.2 \% \\
\text { - APAP } 9.8 \% \pm 4.4 \% \\
\text { - Tramadol/APAP } 15.2 \% \pm 5.7 \% \\
\text { Anti-hyperalgesic effect } \\
\text { (correction for placebo effects) } \\
\text { - Tramadol } 7.4 \% \pm 8.1 \% \\
\text { - APAP } 34.5 \% \pm 14 \% \\
\text { - Tramadol/APAP } 41.1 \% \pm 14.3 \%\end{array}$ \\
\hline Conclusions & $\begin{array}{l}\text { The results suggest that } \\
\text { tramadol-induced anti- } \\
\text { nociception is mediated by } \\
\text { opioid }(\mu) \text { and nonopioid } \\
\text { (inhibition of monoamine } \\
\text { uptake) mechanisms }\end{array}$ & $\begin{array}{l}\text { Alpha }_{2} \text {-adrenoceptor } \\
\text { antagonism reverses tramadol } \\
\text { effects, thus pointing to } \\
\text { significant role of } \\
\text { monoaminergic modulation } \\
\text { and synergy with opioid } \\
\text { antagonism in tramadol } \\
\text { anti-nociception }\end{array}$ & & $\begin{array}{l}\text { Supra-additive effects of the } \\
\text { combination regarding analgesia } \\
\text { and anti-hyperalgesia }\end{array}$ \\
\hline
\end{tabular}

Abbreviations: APAP, paracetamol (acetaminophen); $\mathrm{ED}_{50}$, the dose of a drug that is pharmacologically effective for $50 \%$ of the population exposed to the drug or a $50 \%$ response in a biological system that is exposed to the drug; iv, intravenous. 
In a preclinical model, it has been shown that the nonopioid component in tramadol may enhance its potency ratio relative to morphine in neuropathic pain models. ${ }^{99}$ Tramadol can increase the risk of convulsions in patients who are taking medicinal products reducing the seizure threshold such as bupropion, serotonin reuptake inhibitor antidepressants, tricyclic antidepressants and neuroleptics. In isolated cases there have been reports of serotonin syndrome in a temporal connection with the therapeutic use of tramadol in combination with other serotoninergic medicines such as selective serotonin reuptake inhibitors. ${ }^{100}$ The second component in this fixed-dose combination, paracetamol, appears to act at both central and peripheral pathways, ${ }^{101}$ but its exact mechanism(s) of action has/have yet to be thoroughly elucidated. The maximum recommended adult dose of paracetamol is $4 \mathrm{~g} /$ day. ${ }^{102,103}$ At therapeutic doses, paracetamol is rarely associated with hepatotoxicity. ${ }^{104}$

Complementary pharmacokinetics of tramadol/ paracetamol in combination enhance the probability of effective pain relief (Figure 2) and supra-additive effects of the combination regarding analgesia and anti-hyperalgesia have been demonstrated in a human pain model. ${ }^{33}$ Clinical studies have shown good efficacy and safety of this fixed-dose product for a variety of pain conditions. ${ }^{105,106}$ Details from selected studies can be found in Table 2 .

\section{Mitigation strategies when prescribing high-dose NSAIDs or high-dose paracetamol}

Before high-dose paracetamol or high-dose NSAIDs are considered for patients, mitigation strategies should be undertaken, including the review of patients to verify if they are appropriate candidates for such therapy in light of their comorbidities and co-medications. ${ }^{107}$ Upper gastrointestinal adverse effects can be mitigated by proton pump inhibitors. ${ }^{108-112}$ Patients on long-term high-dose paracetamol or NSAID therapy should be educated as to the potential risks of these drugs, the doses, and the fact that these agents may be contained in a variety of prescription and over-the-counter products. In the USA, this has been called a "do ask, do tell" strategy, where clinicians are encouraged to ask patients about their use of concomitant medications, including over-the-counter products and, by the same token, patients are encouraged to fully disclose to their clinicians all of the drugs they take. ${ }^{113}$ For many patients, it may be appropriate to use a low-dose combination product for maintenance, with occasional NSAIDs to treat breakthrough episodes. An individualized approach to mid- and long-term pain management is required in light of the potential risks and benefits of analgesic agents (Table 4). ${ }^{114}$

The mitigation of adverse events is more than just a matter between clinician and patient. We recommend the use of plain language in labeling over-the-counter products and prescribed medications that contain paracetamol and/or NSAIDs to help patients in monitoring their own daily and cumulative doses. Comprehensive educational efforts are required to alert patients to the dangers of many over-the-counter analgesics and to inform them of appropriate doses and how to calculate them. Many patients consider over-the-counter products "harmless" and may take these agents casually. Patient education should include "do ask, do tell," such that patients understand the importance of discussing with their clinicians all drugs they take.

\section{Current guidelines and pain management in specific populations}

When it comes to pain management, there is no lack of literature, including consensus statements and guidelines.

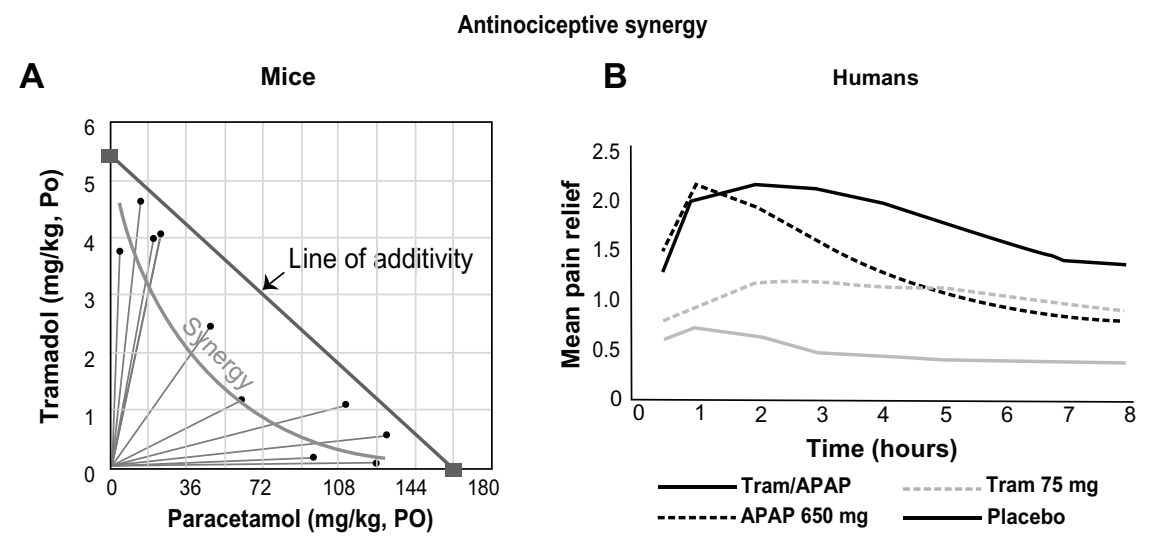

Figure 2 Mean pain relief with (A) tramadol/paracetamol (Tram/APAP) compared with (B) paracetamol $650 \mathrm{mg}$ alone (APAP $650 \mathrm{mg}$ ), tramadol $75 \mathrm{mg}$ alone (Tram $75 \mathrm{mg}$ ), and placebo. Notes: (A) Adapted from Life Sciences, 58(2), Tallarida RJ, Raffa RB, Testing for synergism over a range of fixed ratio drug combinations: replacing the isobologram, PL 23-PL 28, Copyright (1996), with permission from Elsevier. ${ }^{31}$ (B) Adapted from an FDA Executive Summary [web page on the Internet; McNeil background package to the Nonprescription Drug Advisory Committee]. 2002. ${ }^{168}$ 
Table 4 Mitigation strategies that may be useful for patients receiving paracetamol or nonsteroidal anti-inflammatory drugs (NSAIDs) for pain management

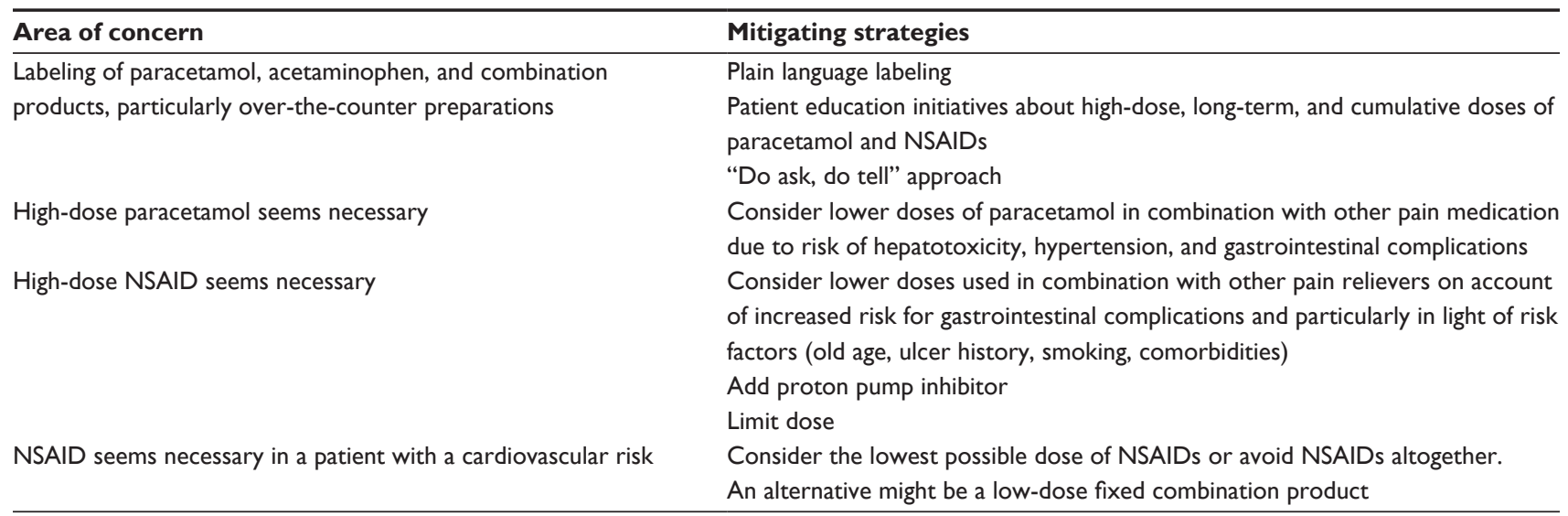

Yet, pain is undertreated. Up to $27 \%$ of people with constant or daily musculoskeletal pain never seek treatment and many people with chronic pain seek medical help for the first time only after a year or more of pain. ${ }^{115}$ It may be inferred that many people feel pain as something they have to live with or that clinicians are unable to treat pain effectively. Between $28 \%$ and $54 \%$ of patients with musculoskeletal pain under medical care do not take any prescription analgesics. ${ }^{115}$ Further, patients may have serious concerns about analgesics; for example, $65 \%-77 \%$ of pain patients considering opioid analgesics have fears of tolerance or addiction. ${ }^{115}$

Many guidelines for the management of pain in specific populations exist. ${ }^{27,29,79,81,116-127}$ These guidelines are largely evidence-based documents, but at times the absence of evidence is construed as the evidence of absence. Important topics in pain management, such as, but not limited to, the transition from acute to chronic pain, are not addressed by the guidelines. In general, the guidelines tend to stress avoidance of adverse events at the expense of efficacy in the treatment of moderate to severe pain. The American Heart Association scientific statement recommends a steppedcare approach to pharmacological therapy for musculoskeletal pain patients with known cardiovascular disease or at risk for ischemic heart disease that emphasizes avoidance of potential risk at the expense of pain relief. ${ }^{29}$

\section{Elderly patients}

Chronic pain is both common and especially challenging to treat in geriatric patients, who often suffer from comorbidities. Chronic pain adversely affects the quality of life, mobility, and mood, and may limit daily activities and social pursuits in patients of all ages, but younger patients may be more resilient or better able to cope with these limitations than older patients. According to the most recent guidelines issued by The American Geriatrics Society, NSAIDs for the treatment of chronic pain should be avoided in patients aged 75 years or older; NSAIDs should be "considered rarely, and with extreme caution, in highly selected individuals. ${ }^{27}$ Paracetamol should be considered as the initial and ongoing therapy of choice except for patients with a known liver disease. The maximum recommended daily dose of paracetamol is $4 \mathrm{~g} / 24$ hours and should not be exceeded. This maximum daily intake must include hidden sources in other medications. All patients with moderate to severe pain, pain-related functional impairment, or diminished quality of life due to pain should be considered for opioid therapy. ${ }^{27}$

\section{Overview on experience with fixed-dose tramadol/paracetamol in the treatment of moderate to severe pain in nonacute conditions: differences to NSAIDs}

NSAIDs are frequently prescribed analgesic agents but recent warnings - including a US Food and Drug Administration labeling proposal that all NSAIDs should be prescribed at the lowest possible doses for the shortest possible duration $^{128}$ - have caused many clinicians to reevaluate these effective painkillers. Recently, new combination analgesic products based on scientifically reasonable design have been introduced to the market to offer effective analgesia with a good risk/benefit ratio. The combination product tramadol/ paracetamol may be an important aid for the treatment of acute and chronic pain syndromes (Table 5).

Pain involving multiple mechanisms, can be safely and effectively treated with combination analgesics, for example, 
Table 5 Comparison of nonsteroidal anti-inflammatory drugs (NSAIDs) with tramadol/paracetamol fixed-dose combination

\begin{tabular}{|c|c|c|}
\hline & Selective and nonselective NSAIDs & Tramadol/paracetamol combination \\
\hline Pain severity & For mild to moderate pain & For moderate to severe pain \\
\hline Clinical application & $\begin{array}{l}\text { Wide, including rheumatic disorders, } \\
\text { headaches, visceral pain }\end{array}$ & $\begin{array}{l}\text { Wide, indicated for symptomatic relief of moderate } \\
\text { to severe pain }\end{array}$ \\
\hline Acute vs chronic pain & Both & Both \\
\hline Neuropathic pain & $\begin{array}{l}\text { No, exclusively for pain related to tissue } \\
\text { damage and/or inflammation }\end{array}$ & $Y_{\text {es }}^{129,130}$ \\
\hline Anti-inflammatory effect & Yes & No \\
\hline Pediatric use & Yes & No \\
\hline Geriatric use & With caution ${ }^{27}$ & May be appropriate 27,150 \\
\hline Use in patients with renal failure & $\mathrm{No}^{163}$ & $\begin{array}{l}\text { Not recommended for severe renal insufficiency } \\
\text { (creatinine clearance }<10 \mathrm{~mL} / \mathrm{min} \text { ) but may be used at } \\
\text { reduced dose in patients with moderate renal insufficiency } \\
\text { (creatinine clearance between } 10 \text { and } 30 \mathrm{~mL} / \mathrm{min} \text { ) } \\
\text { Tramadol is removed only very slowly by hemodialysis or } \\
\text { hemofiltration, so post-dialysis administration to maintain } \\
\text { analgesia is usually not required }\end{array}$ \\
\hline Co-medications & $\begin{array}{l}\text { Caution with diuretics, anticoagulants, } \\
\text { angiotensin-converting-enzyme inhibitors }\end{array}$ & $\begin{array}{l}\text { Caution with other central nervous system depressants, } \\
\text { selective serotonin reuptake inhibitors }{ }^{164}\end{array}$ \\
\hline Use with concomitant opioids & May be synergistic ${ }^{86}$ & Overdose considerations \\
\hline Use with anticonvulsants & Not known & Not known \\
\hline
\end{tabular}

tramadol/paracetamol. ${ }^{129,130}$ However, there are few direct comparative studies of combination products - for instance, codeine/paracetamol versus tramadol/paracetamol. ${ }^{131-133}$

Long-term pain management recommendations often feature NSAIDs as a first-line treatment for rheumatic diseases, ${ }^{134,135}$ with added opioid combination analgesics for flares. ${ }^{136-138}$ A possible new paradigm would be to treat pain first with opioid combination analgesics then use NSAIDs to manage flares. Table 6 summarizes the strengths and weaknesses of NSAIDs versus tramadol/paracetamol fixed-dose combination products.

Recent guidelines for pain management and the position of paracetamol, NSAIDs, and fixed-dose combinations such as tramadol/paracetamol are shown in Table 7.

\section{Consensus statements}

The group arrived at several consensus statements. These follow, grouped by topic.

\section{Pain management}

- There are many reasons why pain management is complex, including the classification of pain, mechanisms, knowledge, individualization, lack of universally accepted guidelines, social and psychological factors, as well as various influences from the health care system itself. Nevertheless, not treating pain is not an option.

- Individualization of treatment in patients suffering from moderate to severe pain should be the ultimate goal of the health care team.

- Pain management guidelines must take into consideration the type of pain, its intensity, the particular patient characteristics, and expected duration of treatment. This requires a multidimensional approach, which creates difficulty in making generalized recommendations.

- Many evidence-based guidelines for pain management are available, but none is universally accepted by all health care providers. These guidelines may benefit

Table 6 Strengths and weakness of tramadol/paracetamol and nonsteroidal anti-inflammatory drugs (NSAIDs)

\begin{tabular}{lll}
\hline & NSAIDs & Tramadol/paracetamol \\
\hline Strengths & - Frequently prescribed & $\bullet$ Recent combination of established analgesics with scientifically \\
& $\bullet$ Ubiquitous & and clinically based rationale \\
& - Gold standard for many conditions: ibuprofen & $\bullet$ Good benefit-risk balance \\
& - Well tolerated short term & $\bullet$ No specific warnings \\
Weaknesses & - Over-the-counter availability & \\
& - Recent warnings & $\bullet$ Combination therapy not well established \\
& - Coadministration with other drugs & $\bullet$ Difficult to differentiate from tramadol immediate release, \\
& & tramadol extended release \\
\end{tabular}


Table 7 Summary of guidelines and recommendations for paracetamol (APAP), nonsteroidal anti-inflammatory drugs (NSAIDs), and combination products such as tramadol (tram)/paracetamol

\begin{tabular}{|c|c|c|c|c|}
\hline Guideline & APAP & NSAIDs & $\begin{array}{l}\text { Combination } \\
\text { (tram/APAP) }\end{array}$ & Comments \\
\hline \multicolumn{5}{|l|}{ Osteoarthritis (OA) } \\
\hline Management of $\mathrm{OA}$ & Ist & (Yes) & Yes & NSAIDS for anti-inflammatory action \\
\hline \multicolumn{5}{|l|}{ Altman overview 117} \\
\hline Early management of OA & Ist & (2nd) & $3 r d$ & Oral NSAIDs at their lowest effective dose; \\
\hline Altman overview 118 & & & & long-term use should be avoided \\
\hline NICE OA guideline ${ }^{81}$ & lst & 2nd with PPI & - & $\begin{array}{l}\text { Oral NSAIDs/COX- } 2 \text { inhibitors should be used } \\
\text { at the lowest effective dose for the shortest } \\
\text { possible period of time }\end{array}$ \\
\hline OARSI guidelines ${ }^{79}$ & Ist & (2nd) & - & $\begin{array}{l}\text { Oral NSAIDs at lowest effective dose; } \\
\text { long-term use should be avoided }\end{array}$ \\
\hline \multicolumn{5}{|l|}{ ACR Guidelines ${ }^{165}$} \\
\hline Hand OA & No & Ist & - & $\begin{array}{l}\text { Topical or oral NSAIDs; topical NSAIDs for } \\
\text { persons } \geq 75 \text { years of age recommended }\end{array}$ \\
\hline Knee OA & lst & 2nd & - & $\begin{array}{l}\text { Health care providers should be aware of the } \\
\text { warnings and precautions associated with topical } \\
\text { and oral NSAIDs }\end{array}$ \\
\hline Hip OA & Ist & 2 nd & - & $\begin{array}{l}\text { Oral NSAIDs; no recommendation on topical } \\
\text { NSAIDs }\end{array}$ \\
\hline \multicolumn{5}{|l|}{ Rheumatoid arthritis (RA) } \\
\hline NICE RA guideline ${ }^{119}$ & Ist & $(2 n d+P P I)$ & $\begin{array}{l}\text { Ist (compound } \\
\text { analgesics in general) }\end{array}$ & $\begin{array}{l}\text { Oral NSAIDs/COX-2 inhibitors should be used } \\
\text { at the lowest effective dose for the shortest } \\
\text { possible period of time }\end{array}$ \\
\hline BSR guidelines for early RA ${ }^{120}$ & $\begin{array}{l}\text { 2nd } \\
\text { (as add-on) }\end{array}$ & (Ist) & 2nd (as add-on) & $\begin{array}{l}\text { Long-term use of NSAIDs at lowest effective } \\
\text { dose. At present, the use of single or compound } \\
\text { analgesics or anti-inflammatory drugs } \\
\text { (including coxibs) has to be settled } \\
\text { with each individual patient } \\
\text { No clear recommendations }\end{array}$ \\
\hline $\begin{array}{l}\text { BSR guidelines for long-term } \\
\text { treatment of } R A^{|2|}\end{array}$ & - & $\begin{array}{l}\text { 2nd as add-on } \\
\text { with PPI }\end{array}$ & - & No clear recommendations \\
\hline $\begin{array}{l}\text { EULAR recommendations } \\
\text { early arthritis }\end{array}$ & - & (Yes) & - & $\begin{array}{l}\text { NSAIDs after careful evaluation of } \\
\text { gastrointestinal, renal, and cardiovascular status }\end{array}$ \\
\hline \multicolumn{5}{|l|}{ Fibromyalgia } \\
\hline $\begin{array}{l}\text { EULAR recommendations } \\
\text { for fibromyalgia }{ }^{123}\end{array}$ & Yes & - & - & Tramadol is one of the analgesics of choice \\
\hline $\begin{array}{l}\text { APS guidelines for } \\
\text { fibromyalgia }^{124}\end{array}$ & No & No & $3 r d$ & $\begin{array}{l}\text { Tricyclic antidepressants first, serotonin } \\
\text { reuptake inhibitors (SSRIs) alone or in } \\
\text { combination with tricyclics second. } \\
\text { Paracetamol not recommended as monotherapy, } \\
\text { only in combination }\end{array}$ \\
\hline \multicolumn{5}{|l|}{ Low back pain } \\
\hline $\begin{array}{l}\text { European guidelines for chronic } \\
\text { nonspecific low back pain }\end{array}$ & - & (Yes) & (Yes) & $\begin{array}{l}\text { NSAIDs should only be used for exacerbations } \\
\text { or short-term periods (up to } 3 \text { months) }\end{array}$ \\
\hline APS/ACP guidelines ${ }^{126}$ & Ist & (lst) & - & $\begin{array}{l}\text { Oral NSAIDs at their lowest effective dose, } \\
\text { for the shortest possible time required }\end{array}$ \\
\hline NICE. Low back pain guideline $e^{127}$ & Ist & $\begin{array}{l}\text { 2nd (+ PPI } \\
\text { for patients } \\
\text { aged }>45 \text { years) }\end{array}$ & - & $\begin{array}{l}\text { Weak opioids and strong opioids are suggested } \\
\text { for more severe pain, but no combinations }\end{array}$ \\
\hline \multicolumn{5}{|l|}{ Musculoskeletal pain } \\
\hline $\begin{array}{l}\text { Schnitzer, guidelines for } \\
\text { chronic musculoskeletal pain"16 }\end{array}$ & & & & $\begin{array}{l}\text { NSAIDs not for long-term use or } \\
\text { in patients with risk factors; second }\end{array}$ \\
\hline Osteoarthritis & Ist & No or 2nd & 2nd & for short-term use \\
\hline
\end{tabular}


Table 7 (Continued)

\begin{tabular}{|c|c|c|c|c|}
\hline Guideline & APAP & NSAIDs & $\begin{array}{l}\text { Combination } \\
\text { (tram/APAP) }\end{array}$ & Comments \\
\hline Low back pain & & 2nd & Ist & $\begin{array}{l}\text { Young, healthy individuals could receive NSAIDs } \\
\text { alone or at a reduced dose combined } \\
\text { with paracetamol/tramadol }\end{array}$ \\
\hline following injury & $\begin{array}{l}\text { 2nd } \\
\text { as add-on }\end{array}$ & $(I s t)$ & $3 r d$ & \\
\hline Rehabilitation & Ist & $\begin{array}{l}\text { Ist for pain in } \\
\text { motion and } \\
\text { for inflammation }\end{array}$ & 2nd as add-on & \\
\hline \multicolumn{5}{|c|}{ Specific patient populations } \\
\hline AGS geriatric guidelines ${ }^{27}$ & Ist & $\begin{array}{l}(2 \mathrm{nd})+\mathrm{PPI} \text { or } \\
\text { misoprostol }\end{array}$ & $(2 n d)$ & $\begin{array}{l}\text { For paracetamol, maximum daily recommended } \\
\text { dosages of } 4 \mathrm{~g} \text { per } 24 \text { hours should not be } \\
\text { exceeded and must include "hidden sources" } \\
\text { Nonselective NSAIDs and COX-2 selective } \\
\text { inhibitors may be considered rarely, and with } \\
\text { extreme caution, in highly selected individuals } \\
\text { All patients with moderate to severe pain, pain- } \\
\text { related functional impairment, or diminished } \\
\text { quality of life due to pain should be considered } \\
\text { for opioid therapy } \\
\text { Maximal safe doses of paracetamol or NSAIDs } \\
\text { should not be exceeded when using fixed-dose } \\
\text { opioid combination agents }\end{array}$ \\
\hline AHA guidelines ${ }^{29}$ & Ist & $(3 r d)$ & - & $\begin{array}{l}\text { NSAIDs at their lowest effective dose }+ \text { ASA } \\
81 \mathrm{mg} \text { and PPI for patients at increased risk } \\
\text { of thrombotic events }\end{array}$ \\
\hline \multicolumn{5}{|l|}{ Neuropathic pain } \\
\hline Dworkin et al ${ }^{166}$ & - & - & - & $\begin{array}{l}\text { Tramadol is recommended as second-line } \\
\text { treatment } \\
\text { Standard treatments such as NSAIDs and } \\
\text { paracetamol have no proven efficacy against } \\
\text { neuropathic pain although they are frequently } \\
\text { prescribed for patients with neuropathic pain }{ }^{167}\end{array}$ \\
\hline
\end{tabular}

Notes: -, not mentioned in guideline; Ist, first-line therapy; 2nd, second-line therapy; 3rd, third-line therapy; NO, not recommended; Yes, recommended but not first-, second-, or third-line recommendation; (Yes), recommended with caution.

Abbreviations: ACR, American College of Rheumatology; ACP, American College of Physicians; AGS, The American Geriatrics Society; AHA, American Heart Association; APS, American Pain Society; ASA, acetylsalicylic acid; BSR, British Society for Rheumatology; COX, cyclooxygenase; coxibs, selective COX-2 inhibitors; EULAR, European League Against Rheumatism; NICE, National Institute for Health and Excellence; PPI, proton pump inhibitor.

by addressing topics such as the chronicity of pain, barriers to treatment, patient preferences influencing pain therapy, and practical clinical considerations. Current guidelines mostly contain strong evidence for pharmacological approaches; however, they would benefit from the addition of considerations related to the evidence or absence of evidence of risks of drugs and inclusion of nonpharmacological treatment options.

\section{The use of NSAIDs and paracetamol in chronic pain management}

- NSAIDs and paracetamol are commonly used and commonly recommended agents for the management of pain and are helpful for many patients. However, they are not without potential risks, especially in the elderly and in patients with renal, gastrointestinal, or cardiovascular disease. High doses and long-term use of NSAIDs to manage moderate to severe pain have been associated with tolerability issues, including serious adverse events.

- Fixed-dose combinations provide a multi-mechanistic analgesic approach. Clinical studies have demonstrated effective management of various types of moderate to severe pain with mostly good tolerability.

- A new approach to managing arthritis-related pain is to consider the long-term use of low-dose combination products for moderate to severe pain, and reserving NSAID use for acute flares related to inflammation. 


\section{The role of fixed-dose combinations in chronic pain management using tramadol/paracetamol as an example}

- Tramadol/Paracetamol may offer distinct advantages in certain patient populations and for certain types of pain, compared with high doses of NSAIDs or paracetamol or when NSAIDs or paracetamol are expected to be used for long durations. However, long-term studies of fixed-dose combinations are required.

- Potential advantages of a fixed-dose tramadol/paracetamol analgesic product include a broader analgesic spectrum, a complementary pharmacokinetic profile, potentially synergistic analgesic effect, greater convenience (possibly resulting in better compliance, thus, improved therapy), and an improved ratio of efficacy to adverse effects.

\section{Conclusion}

Pain management is a global challenge to clinicians and, despite the plethora of evidence-based guidelines, all analgesic options must be individually assessed and weighed for specific risks and benefits in a given patient. Many effective analgesics exist but are associated with adverse events. NSAIDs and paracetamol are effective pain relievers, but recent studies have raised safety concerns, particularly when these agents are used at high doses, long-term, or in special patient populations. Opioid analgesics are effective but are associated with adverse events as well as concerns over tolerance and addiction. Finding an analgesic product that offers both effective pain relief and a good safety profile has led to increasing interest in combination products.

Combination agents may offer analgesic synergy that allows them to provide effective analgesia at reduced doses. However, careful study of combination agents is warranted, as such combination products might also exacerbate side effects. New fixed-dose combination products may offer an improved method of treating the newly recognized multi-mechanistic nature of pain. Studies of fixed-dose combinations such as tramadol/paracetamol for the treatment of chronic pain syndromes are promising, showing safe and effective pain relief with good tolerability and safety profiles.

A new practice paradigm may be to use low-dose paracetamol or fixed-dose combination products, and NSAIDs to manage acute flares. However, further studies are warranted to establish the long-term efficacy and safety of these products.

\section{Acknowledgments}

The meeting was supported by Grünenthal $\mathrm{GmbH}$, Aachen, Germany. Thanks go to Jo Ann LeQuang (LeQ Medical, USA), Birgit Brett (Brett Medical Writing, Germany), and Elke Grosselindemann (Brett Medical Writing, Australia) for editorial assistance and publication coordination. All costs associated with the publication of the manuscript were met by Grünenthal GmbH, Aachen, Germany.

\section{Disclosure}

JV Pergolizzi received consultancy honoraria from Grünenthal GmbH, Baxter, Endo Pharmaceuticals, Purdue Pharma, Janssen, and Hospira. M van de Laar received consultancy honoraria from Merck Netherlands, Pfizer Europa, and Grünenthal $\mathrm{GmbH}$, and speaker honoraria from Pfizer Europa. R Langford has received honoraria and traveling expenses for speaking engagements and consultancy activities from Grünenthal, Janssen-Cilag, Napp/Mundipharma/Purdue, Novartis, Johnson and Johnson/ Ortho-McNeil USA, Pfizer, Bristol Myers Squibb, Javelin Pharmaceuticals, and AstraZeneca. HU Mellinghoff received consultancy honoraria from Grünenthal GmbH. I Moron Merchante received consultancy honoraria from Boehringer Ingelheim, Grünenthal GmbH, Merck Sharp and Dohme Corporation, and Takeda Pharmaceuticals Europe and has received lecture fees from Almirall, Astra-Zeneca, Boehringer Ingelheim, Bristol Myers Squibb, Esteve, Grünenthal GmbH, Eli Lilly and Company, Merck Sharp and Dohme Corporation, Novartis, and Sanofi-Aventis. S Nalamachu has received consultancy honoraria or research grants from the following companies in the past 5 years: Grünenthal $\mathrm{GmbH}$, Johnson and Johnson, Endo Pharmaceuticals, Cephalon, Alphapharma, King Pharmaceuticals, Allergan, ProStakan, and Covidien. J O'Brien received consultancy honoraria from Grünenthal GmbH. S Perrot received consultancy honoraria from Grünenthal GmbH. RB Raffa is a speaker, consultant, and/or basic science investigator for several pharmaceutical companies involved in analgesic research but receives no royalty (cash or otherwise) from the sale of any product; he received consultancy honoraria from Grünenthal $\mathrm{GmbH}$.

\section{List of conference participants}

Participants in the meeting were (authors are indicated with an asterisk): Joseph Pergolizzi* (Johns Hopkins University, Baltimore, Maryland, USA and the Association of Chronic Pain Patients, Houston, Texas, USA); Mart 
van de Laar* (Arthritis Center Twente, Enschede, The Netherlands); Richard Langford* (Anaesthetics Laboratory, St Bartholomew's Hospital, London, UK); Hans-Ulrich Mellinghoff* (Department of Endocrinology, Diabetology and Osteology, Kantonsspital St Gallen, Switzerland); Ignacio Morón Merchante* (Centro de Salud Universitario Goya, Madrid, Spain); Srinivas Nalamachu* (Kansas University Medical Center, Kansas City, Missouri and International Clinic Research, Leawood, Kansas, USA); Joanne O'Brien* (Beaumont Hospital, Dublin, Ireland); Serge Perrot* (Internal Medicine and Therapeutics Department, Hôtel Dieu Hospital, Paris Descartes University, France); Robert B Raffa* (Department of Pharmaceutical Sciences, Temple University School of Pharmacy, Philadelphia, USA); Birgit Brett (Brett Medical Writing, Pulheim, Germany); Karla Schwenke (Medical Affairs, Grünenthal GmbH, Aachen Germany); and Detlef von Zabern (Medical Affairs, Grünenthal GmbH, Aachen, Germany).

\section{References}

1. World Health Organization (WHO). WHO's pain ladder [web page on the Internet]. Geneva: WHO; 2012. Available from: http://www.who. int/cancer/palliative/painladder/en/. Accessed May 12, 2011.

2. WHO. Achieving Balance in National Opioids Control Policy: Guidelines for Assessment. Geneva: WHO; 2000. Available from: whqlibdoc. who.int/hq/2000/who_edm_qsm_2000.4.pdf. Accessed May 13, 2011.

3. WHO. National Cancer Control Programmes: Policies and Managerial Guidelines. 2nd ed. Geneva: WHO; 2002. Available from: http://www. who.int/cancer/media/en/408.pdf. Accessed May 13, 2010.

4. WHO. Cancer Pain Relief with a Guide to Opioid Availability. 2nd ed. Geneva: WHO; 1996. Available from: whqlibdoc.who.int/ publications/9241544821.pdf. Accessed May 12, 2011.

5. Brennan F, Carr DB, Cousins M. Pain management: a fundamental human right. Anesth Analg. 2007;105(1):205-221.

6. Hall JK, Boswell MV. Ethics, law, and pain management as a patient right. Pain Physician. 2009;12(3):499-506.

7. Gwyther L, Brennan F, Harding R. Advancing palliative care as a human right. J Pain Symptom Manage. 2009;38(5):767-774.

8. Fishman SM. Recognizing pain management as a human right: a first step. Anesth Analg. 2007;105(1):8-9.

9. The Societal Impact of Pain. European positioning statement: chronic pain fundamental for European health. International multi-stakeholder platform acknowledges chronic pain as disease in its own-European policy-makers challenged to respond. 2012. Accessed from http:// www.sip-platform.eu/sip-2012.html. Accessed on June 27, 2012.

10. Varrassi G, Müller-Schwefe G, Pergolizzi J, et al. Pharmacological treatment of chronic pain - the need for CHANGE. Curr Med Res Opin. 2010;26(5):1231-1245.

11. Claxton RN, Blackhall L, Weisbord SD, Holley JL. Undertreatment of symptoms in patients on maintenance hemodialysis. J Pain Symptom Manage. 2010;39(2):211-218.

12. Ott BB. Progress in ethical decision making in the care of the dying. Dimens Crit Care Nurs. 2010;29(2):73-80.

13. Fontana JS. The social and political forces affecting prescribing practices for chronic pain. J Prof Nurs. 2008;24(1):30-35.

14. Nathan JI. Chronic pain treatment: a high moral imperative with offsetting personal risks for the physician - a medical student's perspective. Pain Pract. 2010;9(2):155-163.
15. Rejeh N, Vaismoradi M. Perspectives and experiences of elective surgery patients regarding pain management. Nurs Health Sci. 2010; 12(1):67-73.

16. Benyamin RM, Datta S, Falco FJ. A perfect storm in interventional pain management: regulated, but unbalanced. Pain Physician. 2010; 13(2):109-116

17. Ferrell B. Ethical perspectives on pain and suffering. Pain Manage Nur. 2005;63(3):83-90.

18. Rejeh N, Ahmadi F, Mohamadi E, Anoosheh M, Kazemnejad A. Ethical challenges in pain management post-surgery. Nurs Ethics. 2009;16(2):161-171.

19. Macpherson C. Undertreating pain violates ethical principles. $J$ Med Ethics. 2009;35(10):603-606.

20. Ferrell BR. The role of ethics committees in responding to the moral outrage of unrelieved pain. Bioethics Forum. 1997;13(3):11-16.

21. König H, Heider D, Lehnert T, et al; ESEMeD/MHEDEA 2000 investigators. Health status of the advanced elderly in six European countries: results from a representative survey using EQ-5D and SF-12. Health Qual Life Outcomes. 2010;8:143.

22. Breivik H, Collett B, Ventafridda V, Cohen R, Gallacher D. Survey of chronic pain in Europe: prevalence, impact on daily life, and treatment. Eur J Pain. 2006;10(4):287-333.

23. Gerbershagen K, Gerbershagen H, Lutz J, et al. Pain prevalence and risk distribution among inpatients in a German teaching hospital. Clin J Pain. 2009;25(5):431-437.

24. Vlajkovic G, Sindjelic R, Stefanovic I. Ketorolac as a pre-emptive analgesic in retinal detachment surgery: a prospective, randomized clinical trial. Int $J$ Clin Pharmacol Ther. May 2007;45(5): 259-263.

25. Weiner K. Pain Issues: Pain is an Epidemic. Sonora, CA: American Academy of Pain Management; 2001. Available from: http://www. aapainmanage.org/literature/Articles/PainAnEpidemic.pdf. Accessed May 13, 2011

26. Christo PJ, Li S, Gibson SJ, Fine P, Hameed H. Effective treatments for pain in the older patient. Curr Pain Headache Rep. 2011;15(1): $22-34$.

27. American Geriatrics Society Panel on Pharmacological Management of Persistent Pain in Older Persons. Pharmacological management of persistent pain in older persons. J Am Geriatr Soc. 2009;57(8):1331-1346. http:// www.cdc.gov/nchs/data/hus/hus06.pdf. Accessed December 6, 2010.

28. National Center for Health Statistics. Health, United States, 2006: with Chartbook on Trends in the Health of Americans. Hyattsville, MD: National Center for Health Statistics; 2006. Available from: http:// www.cdc.gov/nchs/data/hus/hus06.pdf. Accessed December 6, 2010.

29. Antman EM, Bennett JS, Daugherty A, Furberg C, Roberts H, Taubert KA; American Heart Association. Use of nonsteroidal antiinflammatory drugs: an update for clinicians: a scientific statement from the American Heart Association. Circulation. 2007;115(12): 1634-1642.

30. Raffa RB, Friderichs E, Reimann W, Shank RP, Codd EE, Vaught JL. Opioid and nonopioid components independently contribute to the mechanism of action of tramadol, an 'atypical' opioid analgesic. J Pharmacol Exp Ther. 1992;260(1):275-285.

31. Tallarida RJ, Raffa RB. Testing for synergism over a range of fixed ratio drug combinations: replacing the isobologram. Life Sci. 1996;58(2): PL 23-PL 28.

32. Desmeules JA, Piguet V, Collart L, Dayer P. Contribution of monoaminergic modulation to the analgesic effect of tramadol. $\mathrm{Br} J$ Clin Pharmacol. 1996;41(1):7-12.

33. Filitz J, Ihmsen H, Günther W, et al. Supra-additive effects of tramadol and acetaminophen in a human pain model. Pain. 2008; 136(3):262-270.

34. Baron R, Binder A, Wasner G. Neuropathic pain: diagnosis, pathophysiological mechanisms, and treatment. Lancet Neurol. 2010;9(8): 807-819.

35. Loeser JD, Treede RD. The Kyoto protocol of IASP basic pain terminology. Pain. 2008;137(3):473-477. 
36. International Association for the Study of Pain (IASP). IASP taxonomy [web page on the Internet]. Seattle, WA: IASP; nd [updated May 22, 2012]. http:// www.iasp-pain.org/Content/NavigationMenu/GeneralResourceLinks/ PainDefinitions/default.htm. Accessed June 10, 2012.

37. Woolf CJ; American College of Physicians; American Physiological Society. Pain: moving from symptom control toward mechanism-specific pharmacologic management. Ann Intern Med. 2004;140(6):441-451.

38. Scholz J, Woolf CJ. Can we conquer pain? Nat Neurosci. 2002;5 Suppl:1062-1067.

39. Rainville P, Duncan GH, Price DD, Carrier B, Bushnell MC. Pain affect encoded in human anterior cingulate but not somatosensory cortex. Science. 1997;277(5328):968-971.

40. Woolf CJ, Salter MW. Neuronal plasticity: increasing the gain in pain. Science. 2000;288(5472):1765-1769.

41. Mogil JS, Yu L, Basbaum AI. Pain genes?: natural variation and transgenic mutants. Annu Rev Neurosci. 2000;23:777-811.

42. Gold MS, Gebhart GF. Nociceptor sensitisation in pain pathogenesis. Nat Med. 2010;16(11):1248-1257.

43. Bruehl S. An update on the pathophysiology of complex regional pain syndrome. Anesthesiology. 2010;113(3):713-725.

44. Ren K, Dubner R. Interactions between the immune and nervous systems in pain. Nat Med. 2010;16(11):1267-1276.

45. Graven-Nielsen T, Arednt-Nielsen L. Assessment of mechanisms in localized and widespread musculoskeletal pain. Nat Rev Rheumatol. 2010;6(10):599-606.

46. Holmes A, Williamson O, Hogg M, et al. Predictors of pain severity 3 months after serious injury. Pain Med. 2010;11(7):990-1000.

47. Buvanendran A, Ali A, Stoub TR, Kroin JS, Tuman KJ. Brain activity associated with chronic cancer pain. Pain Phys. 2010;13(5):E337-E342.

48. Perrot S, Javier RM, Marty M, Le Jeunne C, Laroche F; CEDR (Cercle d'Etude de la Douleur en Rhumatologie France), French Rheumatological Society, Pain Study Section. Is there any evidence to support the use of anti-depressants in painful rheumatological conditions? Systematic review of pharmacological and clinical studies Rheumatology (Oxford). 2008;47(8):1117-1123.

49. Chappell AS, Ossanna MJ, Liu-Seifert H, et al. Duloxetine, a centrally acting analgesic, in the treatment of patients with osteoarthritis knee pain: a 13-week, randomized, placebo-controlled trial. Pain. 2009; 146(3):253-260.

50. Jung AC, Staigler T, Sullivan M. The efficacy of selective serotonin reuptake inhibitors for the management of chronic pain. J Gen Intern Med. 1997;12(6):384-389.

51. Mease PJ. Further strategies for treating fibromyalgia: the role of serotonin and norepinephrine reuptake inhibitors. Am J Med. 2009; 122(12 Suppl):S44-S55.

52. Perrot S, Allaert FA, Concas V, Laroche F. "When will I recover?" A national survey on patients' and physicians' expectations concerning the recovery time for acute back pain. Eur Spine J. 2009;18(3): 419-429.

53. Javier RM, Perrot S. Do men and women experience pain differently? What are the implications for the rheumatologist? Joint Bone Spine. 2010;77(3):198-200.

54. Booth M. Opium: A History. New York, NY: Simon and Schuster; 1996.

55. Moulin DE, Clark AJ, Gilron I, et al; Canadian Pain Society. Pharmacological management of chronic neuropathic pain - consensus statement and guidelines from the Canadian Pain Society. Pain Res Manag. 2007;12(1):13-21.

56. Attal N, Cruccu G, Haanpää M, et al; EFNS Task Force. EFNS guidelines on pharmacological treatment of neuropathic pain. Eur J Neurol. 2006;13(11):1153-1169.

57. Finnerup NB, Otto M, McQuay HJ, Jensen TS, Sindrup SH. Algorithm for neuropathic pain treatment: an evidence based proposal. Pain. 2005; 118(3):289-305

58. Dworkin RH, O'Connor AB, Backonja M, et al. Pharmacologic management of neuropathic pain: evidence-based recommendations. Pain. 2007;132(3):237-251.
59. Vargas-Schaffer G. Is the WHO analgesic ladder still valid? Can Fam Physician. 2010;56(6):514-517.

60. Smith HS. Potential analgesic mechanisms of acetaminophen. Pain Physician. 2009;12(1):269-280.

61. American Academy of Pediatrics. Committee on Drugs. Acetaminophen toxicity in children. Pediatrics. 2001;108(4):1020-1024.

62. James LP, Letzig L, Simpson PM, et al. Pharmacokinetics of acetaminophen-protein adducts in adults with acetaminophen overdose and acute liver failure. Drug Metab Dispos. 2009;37(8):1779-1784.

63. Ryder SD, Beckingham IJ. ABC of diseases of liver, pancreas and biliary system. Other causes of parenchymal liver disease. BMJ. 2001;322(7281):290-292.

64. Bronstein AC, Spyker DA, Cantilena LR Jr, Green JL, Rumack BH, Griffin SL. 2008 Annual Report of the American Association of Poison Control Centers' National Poison Data System (NPDS): 26th Annual Report. Clin Toxicol. 2009;47(10):911-1084.

65. Bower WA, Johns M, Margolis HS, Williams IT, Bell BP. Populationbased surveillance for acute liver failure. Am J Gastroenterol. 2007; 102(11):2459-2463.

66. Wilson SL, Poulter NR. The effect of non-steroidal anti-inflammatory drugs and other commonly used non-narcotic analgesics on blood pressure level in adults. J Hypertens. 2006;24(8):1457-1469.

67. Forman JP, Rimm EB, Curhan GC. Frequency of analgesic use and risk of hypertension among men. Arch Intern Med. 2007;167(4):394-399.

68. Sudano I, Flammer AJ, Périat D, et al. Acetaminophen increases blood pressure in patients with coronary artery disease. Circulation. 2010; 122(18):1789-1796.

69. Montgomery B. Does paracetamol cause hypertension? BMJ 2008;336(7654):1190-1191.

70. Montgomery BD. Analgesic use and risk of hypertension: concern about bias. Arch Intern Med. 2007;167(21):2368-2369.

71. White WB, Campbell P. Blood pressure destabilization on nonsteroidal antiinflammatory agents: acetaminophen exposed? Circulation. 2010; 122(18):1779-1781

72. Rao P, Knaus EE. Evolution of nonsteroidal anti-inflammatory drugs (NSAIDs): cyclooxygenase (COX) inhibition and beyond. J Pharm Pharm Sci. 2008;11(2):81s-110s.

73. Vane JR, Botting RM. The mechanism of action of aspirin. Thromb Res. 2003;110(5-6):255-258.

74. Leuppi JD, Schnyder P, Hartmann K, Reinhart WH, Kuhn M. Drug-induced bronchospasm: analysis of 187 spontaneously reported cases. Respiration. 2001;68(4):345-351.

75. van der Linden MW, van der Bij S, Welsing P, Kupiers EJ, Herings RM. The balance between severe cardiovascular and gastrointestinal events among users of selective and non-selective non-steroidal anti-inflammatory drugs. Ann Rheum Dis. 2009;68(5):668-673.

76. Ng SC, Chan FK. NSAID-induced gastrointestinal and cardiovascular injury. Curr Opin Gastroenterol. 2010;26(6):611-617.

77. Lanas A, Perez-Aisa MA, Feu F, et al; Investigators of the Asociación Española de Gastroenterología (AEG). A nationwide study of mortality associated with hospital admission due to severe gastrointestinal events and those associated with nonsteroidal antiinflammatory drug use. Am J Gastroenterol. 2005;100(8):1685-1693.

78. Schjerning Olsen AM, Fosbøl EL, Lindhardsen J, et al. Duration of treatment with nonsteroidal anti-inflammatory drugs and impact on risk of death and recurrent myocardial infarction in patients with prior myocardial infarction: a nationwide cohort study. Circulation. 2011; 123:2226-2235.

79. Zhang W, Moskowitz RW, Nuki G, et al. OARSI recommendations for the management of hip and knee osteoarthritis, Part II: OARSI evidence-based, expert consensus guidelines. Osteoarthritis Cartilage. 2008;16(2):137-162.

80. American College of Rheumatology Ad Hoc Group on Use of Selective and Nonselective Nonsteroidal Antiinflammatory Drugs. Recommendations for use of selective and nonselective nonsteroidal antiinflammatory drugs: an American College of Rheumatology white paper. Arthritis Rheum. 2008;59(8):1058-1073. 
81. National Institute for Health and Excellence (NICE). Osteoarthritis: The Care and Management of Osteoarthritis in Adults. NICE clinical guideline 59. London: NICE; 2008. Available from: http://www.nice.org. uk/nicemedia/pdf/CG59 NICEguideline.pdf. Accessed December 15, 2010.

82. Lanza FL, Chan FK, Quigley EM; Practice Parameters Committee of the American College of Gastroenterology. Guidelines for prevention of NSAID-related ulcer complications. Am J Gastroenterol. 2009; 104(3):728-738.

83. Bhatt DL, Scheiman J, Abraham NS, et al; ACCF Task Force. ACCF/ACG/AHA 2008 expert consensus document on reducing the gastrointestinal risks of antiplatelet therapy and NSAID use: a report of the American College of Cardiology Foundation Task Force on Clinical Expert Consensus Documents. J Am Coll Cardiol. 2008; 52(18):1502-1517.

84. Trelle S, Reichenbach S, Wandel S, et al. Cardiovascular safety of non-steroidal anti-inflammatory drugs: network meta-analysis. $B M J$. 2011;342:c7086

85. Desai JC, Sanyal SM, Goo T, et al. Primary prevention of adverse gastroduodenal effects from short-term use of non-steroidal antiinflammatory drugs by omeprazole $20 \mathrm{mg}$ in healthy subjects: a randomized, double-blind, placebo-controlled study. Dig Dis Sci. 2008;53(8):2059-2065.

86. Raffa RB. Pharmacology of oral combination analgesics: rational therapy for pain. J Clin Pharm Ther. 2001;26(4):257-264.

87. Raffa RB, Pergolizzi JV, Segarnick DJ, Tallarida RJ. Oxycodone combinations for pain relief. Drugs Today (Barc). 2010;46(6): 379-398.

88. Tallarida RJ, Cowan A, Raffa RB. Antinociceptive synergy, additivity, and subaddivity with combinations of oral glucosamine plus nonopioid analgesics in mice. J Pharmacol Exp Ther. 2003;307(2): 699-704

89. Tallarida RJ. Drug synergism: its detection and applications. J Pharmacol Exp Ther. 2001;298(3):865-872.

90. Tallarida RJ, Stone DJ Jr, Raffa RB. Efficient designs for studying synergistic drug combinations. Life Sci. 1997;61(26):PL 417-PL 425.

91. Tallarida RJ. Interactions between drugs and occupied receptors. Pharmacol Ther. 2007;113(1):197-209.

92. Tallarida RJ. An overview of drug combination analysis with isobolograms. Perspectives in pharmacology. J Pharmacol Exp Ther. 2006;319(1):1-7.

93. Loewe L. Antagonisms and antagonists. Pharmacol Rev. 1957;9(2): 237-242.

94. Loewe $\mathrm{S}$. The problem of synergism and antagonism of combined drugs. Arzneimittelforschung. 1953;3(6):285-290.

95. Tallarida RJ. Drug Synergism and Dose-Effect Data Analysis. Boca Raton, FL: CRC/Chapman-Hall; 2000.

96. Grabovsky Y, Tallardia RJ. Isobologrphic analysis for combinations of full and partial agonists: curved isoboles. J Pharmacol Exp Ther. 2004;310(3):981-986.

97. Braverman AS, Tallarida RJ, Ruggieri MR Sr. The use of occupation isoboles for analysis of a response mediated by two receptors: M2 and M3 muscarinic receptor subtype-induced mouse stomach contraction. J Pharmacol Exp Ther. 2008;325(3):954-960.

98. Ide S, Minami M, Ishihara K, Uhl GR, Sora I, Ikeda K. Mu opioid receptor-dependent and independent components in effects of tramadol. Neuropharmacology. 2006;51(3):651-658.

99. Christoph T, Kögel B, Strassburger W, Schug SA. Tramadol has a better potency ratio relative to morphine in neuropathic than in nociceptive pain models. Drugs R D. 2007;8(1):51-57.

100. Zaldiar ${ }^{\circledR}$ product monograph. Aachen:Gruenenthal GmbH: 2002. Available from: http://www.zaldiar.com/zal/en_EN/pdf/zaldiar_product_monograph.pdf. Accessed June 27, 2012.

101. Bonnefont J, Courade JP, Alloui A, Eschalier A. Antinociceptive mechanism of action of paracetamol. Drugs. 2003;63 Spec No 2: $1-4$. French.
102. Hawton K, Ware C, Mistry H, et al. Paracetamol self-poisoning: characteristics, prevention and harm reduction. Br J Psychiatry. 1996; 168(1):43-48.

103. Kuffner EK, Dart RC, Bogdan GM, Hill RE, Casper E, Darton L. Effect of maximal daily doses of acetaminophen on the liver of alcoholic patients: a randomized, double-blind, placebo-controlled trial. Arch Intern Med. 2001;161(18):2247-2252.

104. Gregoire N, Hovsepian L, Gualano V, Evene E, Dufour G, Gendron A. Safety and pharmacokinetics of paracetamol following intravenous administration of $5 \mathrm{~g}$ during the first $24 \mathrm{~h}$ with a 2 -g starting dose. Clin Pharmacol Ther. 2007;81(3):401-405.

105. McClellan K, Scott LJ. Tramadol/paracetamol. Drugs. 2003;63(11): 1079-1086.

106. Dhillon S. Tramadol/paracetamol fixed-dose combination: a review of its use in the management of moderate to severe pain. Clin Drug Investig. 2010;30(10):711-738.

107. Scheiman JM. Balancing risks and benefits of cyclooxygenase- 2 selective nonsteroidal anti-inflammatory drugs. Gastroenterol Clin N Am. 2009;38(2):305-314.

108. van der Linden MW, Gaugris S, Kuipers EJ, et al. COX-2 inhibitors: complex association with lower risk of hospitalization for gastrointestinal events compared to traditional NSAIDs plus proton pump inhibitors. Pharmacoepidemiol Drug Saf. 2009;18(10):880-890.

109. Rahme E, Barkun AN, Toubouti Y, Scalera A, Rochon S, Lelorier J. Do proton-pump inhibitors confer additional gastrointesntinal protection in patients given celecoxib? Arthritis Rheum. 2007;57(5):748-755.

110. Laine L. Proton pump inhibitor co-therapy with nonsteroidal antiinflammatory drugs - nice or necessary? Rev Gastroenterol Disord. 2004;4 Supp1 4:S33-S41.

111. Chan FK, Wong VW, Suen BY, et al. Combination of a cyclooxygenase-2 inhibitor and a proton-pump inhibitor for prevention of recurrent ulcer bleeding in patients at very high risk: a double-blind, randomised trial. Lancet. 2007;369(9573):1621-1626.

112. Vonkeman HE, Fernandes RW, van der Palen J, van Roon EN, van de Laar MA. Proton-pump inhibitors are associated with a reduced risk for bleeding and perforated gastroduodenal ulcers attributable to non-steroidal anti-inflammatory drugs: a nested case-control study. Arthritis Res Ther. 2007;9(3):R52.

113. US Food and Drug Administration (FDA). Safe Use Initiative: collaborating to reduce preventable harm from medications [web page on the Internet]. Silver Spring, MD: FDA; nd [updated April 26, 2012]. Available from: http://www.fda.gov/Drugs/DrugSafety/ SafeUseInitiative/default.htm. Accessed May 13, 2011.

114. Wong VW, Chan FK. Cycolooxygenase-2 inhibitors in patients with high gastrointestinal risk: are we there yet? J Gastroenterol. 2009;44 Suppl 19:53-56.

115. Woolf AD, Zeidler H, Haglund U, et al. Musculoskeletal pain in Europe: its impact and a comparison of population and medical perceptions of treatment in eight European countries. Ann Rheum Dis. 2004;63(4):342-347.

116. Schnitzer TJ. Update on guidelines for the treatment of chronic musculoskeletal pain. Clin Rheumatol. 2006;25 Suppl 1:S22-S29.

117. Altman RD. Practical considerations for the pharmacologic management of osteoarthritis. Am J Manag Care. 2009;15(8 Suppl):S236-S243.

118. Altman RD. Early management of osteoarthritis. Am J Manag Care. 2010;16 Suppl Management:S41-S47.

119. NICE. RheumatoidArthritis: The Management of RheumatoidArthritis in Adults. NICE clinical guideline 79. London: NICE; 2009. Available from: http://www.nice.org.uk/nicemedia/live/12131/43327/43327.pdf. Accessed December 18, 2010.

120. Luqmani R, Hennell S, Estrach C, et al; British Society for Rheumatology; British Health Professionals in Rheumatology Standards, Guidelines and Audit Working Group. British Society for Rheumatology and british health professionals in Rheumatology guideline for the management of rheumatoid arthritis (the first two years). Rheumatology (Oxford). 2006;45(9):1167-1169. 
121. Luqmani R, Hennell S, Estrach C, et al; British Society for Rheumatology; British Health Professionals in Rheumatology Standards, Guidelines and Audit Working Group. British Society for Rheumatology and British Health Professionals in Rheumatology guideline for the management of rheumatoid arthritis (after the first 2 years). Rheumatology (Oxford). 2009;48(4):436-439.

122. Combe B, Landewe R, Lukas C, et al. EULAR recommendations for the management of early arthritis: report of a task force of the European Standing Committee for International Clinical Studies Including Therapeutics (ESCISIT). Ann Rheum Dis. 2007;66(1):34-45.

123. Carville SF, Arendt-Nielsen S, Bliddal H, et al; EULAR. EULAR evidence-based recommendations for the management of fibromyalgia syndrome. Ann Rheum Dis. 2008;67(4):536-541.

124. Burckhardt C, Goldenberg D, Crofford L, et al. Guideline for the Management of Fibromyalgia Syndrome Pain in Adults and Children. Clinical practice guideline 4. 2005. Available from: http://www.ampainsoc.org/library/pt_fibromyalgia.htm. Accessed December 18, 2010.

125. Airaksinen O, Brox JI, Cedraschi C, et al; COST B13 Working Group on Guidelines for Chronic Low Back Pain. Chapter 4. European guidelines for the management of chronic nonspecific low back pain. Eur Spine J. 2006;15 Suppl 2:S192-S300.

126. Chou R, Hoyt-Huffman L; American Pain Society; American College of Physicians. Medications for acute and chronic low back pain: a review of the evidence for an American Pain Society/American College of Physicians clinical practice guideline. Ann Intern Med. 2007;147(7):505-514.

127. NICE. Low Back Pain: Early Management of Persistent NonSpecific Low Back Pain. NICE clinical guideline 88. London: NICE; 2009. Available from: http://www.nice.org.uk/nicemedia/ live/11887/44343/44343.pdf. Accessed December 18, 2010.

128. FDA. Proposed NSAID package insert labeling template 1 . Silver Spring, MD: FDA; nd. Available from: http://www.fda.gov/ downloads/Drugs/DrugSafety/PostmarketDrugSafetyInformation forPatientsandProviders/ucm106230.pdf. Accessed December 18 , 2010.

129. Ko SH, Kwon HS, Yu JM, et al. Comparison of the efficacy and safety of tramadol/acetaminophen combination therapy and gabapentin in the treatment of painful diabetic neuropathy. Diabet Med. 2010;27(9):1033-1040.

130. Freeman R, Raskin P, Hewitt DJ, et al; CAPSS-237 Study Group. Randomized study of tramadol/acetaminophen versus placebo in painful diabetic peripheral neuropathy. Curr Med Res Opin. 2007; 23(1):147-161

131. Mullican WS, Lacy JR; TRAMAP-ANAG-006 Study Group. Tramadol/acetaminophen combination tablets and codeine/ acetaminophen combination capsules for the management of chronic pain: a comparative trial. Clin Ther. 2001;23(9):1429-1445.

132. Smith AB, Ravikumar TS, Kamin M, Jordan D, Xiang J, Rosenthal N; CAPSS-115 Study Group. Combination tramadol plus acetaminophen for postsurgical pain. Am J Surg. 2004;187(4):521-527.

133. Pickering G, Estrade M, Dubray C. Comparative trial of tramadol/ paracetamol and codeine/paracetamol combination tablets on the vigilance of healthy volunteers. Fundam Clin Pharmacol. 2005;19(6): 707-711.

134. Richmond J, Hunter D, Irrgang J, et al; American Academy of Orthopaedic Surgeons. American Academy of Orthopaedic Surgeons clinical practice guideline on the treatment of osteoarthritis (OA) of the knee. J Bone Joint Surg Am. 2010;92(4):990-993.

135. Cibulka MT, White DM, Woehrle J, et al. Hip pain and mobility deficits - hip osteoarthritis: clinical practice guidelines linked to the international classification of functioning, disability, and health from the orthopaedic section of the American Physical Therapy Association. J Orthop Sports Phys Ther. Apr 2009;39(4):A1-A25.

136. Schnitzer TJ; American College of Rheumatology. Update of ACR guidelines for osteoarthritis: role of the coxibs. J Pain Symptom Manage. 2002;23(4 Suppl):S24-S30; discussion S31-S34.
137. Conaghan PG, Dickson J, Grant RL. Care and management of osteoarthritis in adults: summary of NICE guidance. BMJ. 2008; 336(7642):502-503.

138. Zhang W, Doherty M, Arden N, et al; EULAR Standing Committee for International Clinical Studies Including Therapeutics (ESCISIT). EULAR evidence based recommendations for the management of hip osteoarthritis: report of a task force of the EULAR Standing Committee for International Clinical Studies Including Therapeutics (ESCISIT). Ann Rheum Dis. 2005;64(5):669-681.

139. Fricke JR Jr, Karim R, Jordan D, Rosenthal N. A double-blind, singledose comparison of the analgesic efficacy of tramadol/acetaminophen combination tablets, hydrocodone/acetaminophen combination tablets, and placebo after oral surgery. Clin Ther. 2002;24(6): 953-968.

140. Macleod AG, Ashford B, Voltz M, et al. Paracetamol versus paracetamol-codeine in the treatment of post-operative dental pain: a randomized, double-blind, prospective trial. Aust Den J. 2002;47(2): $147-151$

141. Edwards JE, McQuay HJ, Moore RA. Combination analgesic efficacy: individual patient data meta-analysis of single-dose oral tramadol plus acetaminophen in acute postoperative pain. J Pain Symptom Manage. 2002;23(2):121-130

142. Jung YS, Kim DK, Kim MK, Kim HJ, Cha IH, Lee EW. Onset of analgesia and analgesic efficacy of tramadol/acetaminophen and codeine/acetaminophen/ibuprofen in acute postoperative pain: a single-center, single-dose, randomized, active-controlled, parallelgroup study in a dental surgery pain model. Clin Ther. 2004;26(7): 1037-1045

143. Litkowski LJ, Christensen SE, Adamson DN, Van Dyke T, Han SH, Newman KB. Analgesic efficacy and tolerability of oxycodone $5 \mathrm{mg} /$ ibuprofen $400 \mathrm{mg}$ compared with those of oxycodone $5 \mathrm{mg} /$ acetaminophen $325 \mathrm{mg}$ and hydrocodone $7.5 \mathrm{mg} /$ acetaminophen $500 \mathrm{mg}$ in patients with moderate to severe postoperative pain: a randomized, double-blind, placebo-controlled, single-dose, parallel-group study in a dental pain model. Clin Ther. 2005;27(4): 418-429.

144. Daniels SE, Goulder MA, Aspley S, Reader S. A randomised, fiveparallel-group, placebo-controlled trial comparing the efficacy and tolerability of analgesic combinations including a novel single-tablet combination of ibuprofen/paracetamol for postoperative dental pain. Pain. 2011;152(3):632-642.

145. White PF, Joshi GP, Carpenter RL, Fragen RJ. A comparison of oral ketorolac and hydrocodone-acetaminophen for analgesia after ambulatory surgery: arhroscopy versus laparoscopic tubal ligation. Anesth Analg. 1997;85(1):37-43.

146. Palangio M, Damask MJ, Morris E, et al. Combination hydrocodone and ibuprofen versus combination codeine and acetaminophen for the treatment of chronic pain. Clin Ther. 2000;22(7):879-892.

147. Sniezek PJ, Brodland DG, Zitelli JA. A randomized controlled trial comparing acetaminophen, acetaminophen and ibuprofen, and acetaminophen and codeine for postoperative pain relief after Mohs surgery and cutaneous reconstruction. Dermatol Surg. 2011;37(7): 1007-1013.

148. Rawal N, Macquaire V, Catalá E, Berti M, Costa R, Wietlisbach M. Tramadol/paracetamol combination tablet for postoperative pain following ambulatory hand surgery: a double-blind, double-dummy, randomized, parallel-group trial. J Pain Res. 2011;4:103-110.

149. Serrie A, Ganry H, Creuzé A, Schatz B. Epidemiological data, efficacy and safety on a fixed combination of paracetamol (325 mg) and tramadol $(37.5 \mathrm{mg})$ in the treatment of moderate to severe pain, in general practice (ELZA survey: Efficacité et ToLerance de ZAldier ${ }^{\circledR}$ ). Journal of Applied Therapeutic Research. 2011;8(1):3-5.

150. Mejjad O, Serrie A, Ganry H. Epidemiological data, efficacy and safety of a paracetamol-tramadol fixed combination in the treatment of moderate-to-severe pain. SALZA: a post-marketing study in general practice. Curr Med Res Opin. 2011;27(5):1013-1020. 
151. Emkey R, Rosenthal N, Wu SC, Jordan D, Kamin M; CAPSS-114 Study Group. Efficacy and safety of tramadol/acetaminophen tablets (Ultracet) as add-on therapy for osteoarthritis pain in subjects receiving a COX-2 nonsteroidal antiinflammatory drug: a multicenter, randomized, double-blind, placebo-controlled trial. J Rheumatol. 2004;31(1):150-156.

152. Corsinovi L, Martinelli E, Fonte G, et al. Efficacy of oxycodone/ acetaminophen and codeine/acetaminophen vs conventional therapy in elderly women with persistent, moderate to severe osteoarthritisrelated pain. Arch Gerontol Geriatr. 2009;49(3):387-382.

153. Pareek A, Chandurkar N, Sharma VD, Desai M, Kini S, Bartakke G. A randomized, multicentric, comparative evaluation of aceclofenacparacetamol combination with aceclofenac alone in Indian patients with osteoarthritis flare-up. Expert Opin Pharmacother. 2009;10(5): 727-735.

154. Pareek A, Chandurkar N, Ambade R, Chandanwale A, Bartakke G. Efficacy and safety of etodolac-paracetamol fixed dose combination in patients with knee osteoarthritis flare-up: a randomized, double-blind comparative evaluation. Clin J Pain. 2010;26(7): 561-566.

155. Doherty M, Hawkey C, Goulder M, et al. A randomised controlled trial of ibuprofen, paracetamol or a combination tablet of ibuprofen/ paracetamol in community-derived people with knee pain. Ann Rheum Dis. 2011;70(9):1534-1541.

156. Conaghan PG, O’Brien CM, Wilson M, Schofield JP. Transdermal buprenorphine plus oral paracetamol vs an oral codeine-paracetamol combination for osteoarthritis of hip and/or knee: a randomised trial. Osteoarthritis Cartilage. 2011;19(8):930-938.

157. Palangio M, Morris E, Doyle RT Jr, Dornseif BE, Valente TJ. Combination hydrocodone and ibuprofen versus combination oxycodone and acetaminophen in the treatment of moderate or severe acute low back pain. Clin Ther. 2002;24(1):87-99.

158. Ruoff GE, Rosenthal N, Jordan D, Karim R, Kamin M; Protocol CAPSS-112 Study Group. Tramadol/acetaminophen combination tablets for the treatment of chronic lower back pain: a multicenter, randomized, double-blind, placebo-controlled outpatient study. Clin Ther. 2003;25(4):1123-1141.
159. Perrot S, Krause D, Crozes P, Naïm C; GRTF-ZAL-1 Study Group. Efficacy and tolerability of paracetamol/tramadol (325 mg/37.5 mg) monotherapy in patients with subacute low back pain: a multicenter, randomized, double-blind, parallel-group, 10-day treatment study. Clin Ther. 2006;28(10):1592-1606.

160. Bennett RM, Kamin M, Karim R, Rosenthal N. Tramadol and acetaminophen combination tablets in the treatment of fiobromyalgia pain: a double-blind, randomized, placebo-controlled study. Am J Med. 2003;114(7):537-545.

161. Lee EY, Lee EB, Park BJ, et al. Tramadol 37.5-mg/acetaminophen 325-mg combination tablets added to regular therapy for rheumatoid arthritis pain: a 1-week, randomized, double-blind, placebo-controlled trial. Clin Ther. 2006;28(12):2052-2060.

162. Raffaeli W, Pari C, Corvetta A, et al. Oxycodone/acetaminophen at low dosage: an alternative pain treatment for patients with rheumatoid arthritis. J Opioid Manag. 2010;6(1):40-46.

163. Graham GG, Graham RI, Day RO. Comparative analgesia, cardiovascular, and renal effects of celecoxib, rofecoxib and acetaminophen (paracetamol). Curr Pharm Des. 2002;8(12):1063-1075.

164. Hersh EV, Pinto A, Moore PA. Adverse drug interactions involving common prescription and over-the-counter analgesic agents. Clin Ther. 2007;29 Suppl:2477-2497.

165. Hochberg MC, Altman RD, Toupin April K, et al. American College of Rheumatology 2012 recommendations for the use of nonpharmacologic and pharmacologic therapies in osteoarthritis of the hand, hip, and knee. Arthritis Care and Research. 2012;64(4):465-474.

166. Dworkin RH, O'Connor AB, Audette J, et al. Recommendations for the pharmacological management of neuropathic pain: an overview and literature update. Mayo Clin Proc. 2010;85(3 Suppl):S3-S14

167. Haanpää M, Backonja MM, Bennett M, et al. Assessment of neuropathic pain in primary care. Am J Med. 2009;122(10 Suppl): S13-S21.

168. FDA. Executive summary [web page on the Internet; McNeil background package to the Nonprescription Drug Advisory Committee]. Silver Spring, MD: FDA; 2002. Available from: http://www.fda.gov/ ohrms/dockets/ac/02/briefing/3882B1_13_McNeil-Acetaminophen. htm\#_Toc18717551. Accessed May 13, 2011.
Journal of Pain Research

\section{Publish your work in this journal}

The Journal of Pain Research is an international, peer-reviewed, open access, online journal that welcomes laboratory and clinical findings in the fields of pain research and the prevention and management of pain. Original research, reviews, symposium reports, hypothesis formation and commentaries are all considered for publication.

\section{Dovepress}

The manuscript management system is completely online and includes a very quick and fair peer-review system, which is all easy to use. Visit http://www.dovepress.com/testimonials.php to read real quotes from published authors. 